\title{
NPD und AfD als Wiedergänger des Nationalsozialismus und Nationalismus? Eine Bildanalyse von Magazincovern
}

\author{
Lorenz Klumpp
}

Online publiziert: 4. August 2020

(C) Der/die Autor(en) 2020

Zusammenfassung Im Zuge des Erscheinens und der Etablierung der Alternative für Deutschland (AfD) widmen sich zahlreiche Studien ihrem Aufstieg, ihren Kommunikationsstrategien sowie der medialen Berichterstattung über die Partei. Diese Studien beziehen sich überwiegend auf textuelle Botschaften. Da visuelle Medien jedoch allgegenwärtig sind und unser Verständnis bzw. unsere Interpretationen von Politik beeinflussen, gilt es, politische Sachverhalte stärker als bislang mittels bildbezogener Ansätze zu reflektieren. Im theoretischen Teil wird dies unter Bezugnahme auf die Konzepte der politischen Vision und der visuellen Politik begründet. Empirisch wird anschließend mittels ikonographisch-ikonologischer Analysen die Frage beantwortet, wie das Nachrichtenmagazin Der Spiegel die Nationaldemokratische Partei Deutschlands (NPD) und die AfD in ihren Aufstiegs- bzw. Etablierungsphasen auf seinen Titelbildern visualisiert. Ein Vergleich von Darstellungen jener Parteien, die in der Geschichte der Bundesrepublik im rechten Spektrum erfolgreich waren, erlaubt es, Kontinuitäten im visuellen medialen Framing dieser Akteure herauszustellen.

Die explorative Analyse zeigt, dass Der Spiegel auf seinen Titelbildern sowohl in Bezug auf die NPD als auch auf die AfD mehrfach auf Vergleiche zum Nationalsozialismus wie auch zum übersteigerten Nationalismus setzt. Außerdem steht der dynamische Aufstieg beider Parteien im Mittelpunkt. Die visuellen Frames werden vor dem Hintergrund ihrer potentiellen Bedeutungen für den Diskurs kritisch erörtert. Außerdem werden Wege für die Ausweitung der präsentierten Bildanalysen aufgezeigt.

Der Aufsatz entstand im Rahmen des Projekts „Visualisierung der Demokratie - ViDe(m)o“, das innerhalb des Bayerischen Forschungsverbunds „ForDemocracy“ vom Bayerischen Staatsministerium für Wissenschaft und Kunst gefördert wird.

L. Klumpp, M.A. $(\bowtie)$

Professur für Methoden der empirischen Sozialforschung, Universität Passau,

Dr.-Hans-Kapfinger-Straße 14b, Passau, Deutschland

E-Mail: lorenz.klumpp@uni-passau.de 


\title{
NPD and AfD as revenants of Nazism and Nationalism? A visual analysis of magazine covers
}

\begin{abstract}
Numerous studies deal with the rise of right-wing populist parties, like the Alternative für Deutschland (AfD) in Germany, as well as with their communication strategies and the representation of these actors in the mass media. But only few are interested in the disseminated visual imagery. As visual media are omnipresent in our everyday life and therefore shape our understanding and interpretation of politics, this paper argues that social science needs to draw more attention to visual approaches. In a first step, this will be outlined on a theoretical level with a special focus on the concepts of political vision and visual politics. By drawing on an iconographic-iconological approach the paper will then answer the question of how the German weekly news magazine Der Spiegel visualised the National Democratic Party of Germany (NPD) and the AfD during their initial respectively established phases. The comparison of the visualisations shows several continuities in the visual framing of the two right-wing parties that were successful on a national level.

The exploratory analysis reveals that, in both cases, Der Spiegel puts emphasis on analogies to Nazism and exaggerated nationalism. Additionally, we can observe a focus on the dynamic rise of the two parties. The visual frames are discussed against the background of their potential meaning for public discourse. Finally, ways of expanding the visual analysis in this field of research are pointed out.
\end{abstract}

\section{Einleitung}

Der in den letzten Jahren zu beobachtende Aufstieg von rechtspopulistischen Parteien wird sowohl in der öffentlichen als auch in der wissenschaftlichen Debatte als Bedrohung für die liberale Demokratie wahrgenommen (Rummens 2017; Ulrich 2017). Parteien rechts der Union werden in Deutschland grundsätzlich mit besonderer Skepsis betrachtet, da sie geschichtlich bedingt im ,Widerspruch zu einer deutschen Mehrheitskultur" (Jaschke 2016, S. 121) stehen. Dies galt lange als Erklärung dafür, dass sich hierzulande keine rechtspopulistische Partei langfristig auf nationaler Ebene etablieren konnte. Dies hat sich im letzten Jahrzehnt mit dem Aufstieg bzw. der Etablierung der Alternative für Deutschland (AfD) geändert. Im Zuge dessen sind zahlreiche Publikationen zur politischen Kommunikation seitens der AfD vorgelegt worden, etwa zu den Inhalten der Parteiprogramme (Arzheimer 2015; Franzmann und Lewandowsky 2020), zu ihrer Nutzung von sozialen Medien (Arzheimer 2015; Stier et al. 2017) sowie zu populistischen Erzählstrategien (Gadinger und Simon 2019). Parallel dazu hat auch die Medienberichterstattung über die AfD das Interesse der Forschung geweckt (Bey und Wamper 2018; Ludwig und Wamper 2017). Zum Teil werden Handreichungen für Journalist*innen formuliert (Gäbler 2017, 2018). Was jedoch die visuelle Darstellung rechtspopulistischer Akteure in den Medien betrifft, hat lediglich Herkman (2019) eine Studie vorgelegt. Er vergleicht Karikaturen populistischer Parteiführungen in den skandinavischen Ländern. Vor dem Hintergrund dieser Forschungslücke im deutschen Kontext nimmt der 
vorliegende Beitrag Herkmans Arbeit als Anregung für eine Auseinandersetzung mit massenmedialen Visualisierungen der AfD.

Davon ausgehend, dass massenmedial verbreitete Bilder zur Sinnkonstruktion politischer Wirklichkeit beitragen (Aiello und Parry 2020; Müller 1999), wird hier der Frage nachgegangen, entlang welcher visueller Frames (Geise et al. 2013) das Nachrichtenmagazin Der Spiegel auf seinen Covern über die AfD berichtet. Um mögliche Kontinuitäten im Sinne eines Bilderkanons (Paul 2008) hinsichtlich der visuellen Darstellungen der heute wieder breit diskutierten Gefahr von rechts aufdecken zu können, werden zudem Cover aus der erfolgreichen Anfangsphase der NPD zwischen 1964 und 1969 zum Vergleich herangezogen. Historisch gesehen war sie bis zum Erscheinen der AfD die einzige überregional erfolgreiche Partei rechts der Union (Decker und Lewandowsky 2017, S. 32; Werz 2013, S. 422), weshalb sich der Vergleich der Visualisierungen in einem politischen Magazin anbietet. Entscheidend für diesen Beitrag sind die in Bezug auf die beiden rechten Parteien präsentierten Deutungsangebote. ${ }^{1}$

Im Folgenden werden zunächst die Konzepte der politischen Vision und der visuellen Politik vorgestellt. Sie fußen zum einen auf einem kommunikationszentrierten Verständnis von Politik, das die Medien als Mittler zwischen Politik und Bürger*innen in den Mittelpunkt stellt. Zum anderen basieren sie auf der Annahme, dass diese Mittlerrolle maßgeblich von ikonischen Elementen geprägt ist (Hofmann 2009, 1999b; Müller 1999). Mittels der ikonographisch-ikonologischen Analyse nach Winfried Marotzki und Katja Stoetzer (2006) - einem zunächst deskriptiven und anschließend interpretativen Verfahren, mit dem sich Sinnkonstruktionen offenlegen und mögliche Bildbedeutungen für den Diskurs erschließen lassen - werden sodann visuelle Frames in der Berichterstattung über die NPD und die AfD herausgearbeitet.

\section{Theoretische Überlegungen}

\subsection{Ein visuelles Verständnis von Politik}

Das für diesen Beitrag relevante Verständnis von visueller Politik ist vor dem Hintergrund einer Debatte in der politischen Kulturforschung zu sehen, in der Karl Rohe dafür plädierte, politische Kultur stärker als Muster von Vor- und nicht ausschließlich von Einstellungen zu konzeptualisieren. Er unterscheidet zwischen zwei Ebenen, der ,politischen Sozialkultur“ und der ,politischen Deutungskultur“, deren Austauschverhältnis ,ein konstitutives Wesensmoment von politischer Kultur“

\footnotetext{
1 Ungeachtet dessen sind Unterschiede in der theoretischen Betrachtung und Einordnung hinsichtlich der beiden Parteien sowie zeitgenössische politische Unterschiede zu berücksichtigen. So wurde die NPD damals wie heute überwiegend als rechtsextremistische Partei eingestuft (Dudek und Jaschke 1984; Kühnl 1967). Im Gegensatz zur Kategorisierung der AfD spielte der Begriff des Populismus in der wissenschaftlichen Debatte um die ,alte NPD“ (Pfahl-Traughber 2019, S. 57 ff.) keine Rolle. Für die heutigen Erscheinungsformen des Populismus sowie terminologische Abgrenzungen zwischen Rechtspopulismus und Rechtsextremismus vgl. Decker und Lewandowsky (2017), Jesse und Panreck (2017) sowie Priester (2016).
} 
(Rohe 1987, S. 42) darstellt. Während er unter politischer Sozialkultur die in spezifischen Bevölkerungsgruppen mehr oder weniger verfestigten Einstellungen versteht - also den Bereich, der methodisch klassischerweise durch Umfragen abgedeckt wird - bezeichnet er politische Deutungskultur als „kulturelle Aktivitäten, Prozesse und Produkte in jenem und aus jenem Bereich [...], den wir aus einem traditionellen Vorverständnis heraus den ,Kulturbereich“ der Gesellschaft nennen“ (ebd.). Für die Erforschung der politischen Deutungskultur sind hermeneutische Ansätze zentral. „Denn politische Soziokultur ist nicht etwas, was in einer bestimmten Lebensphase ein für allemal ,einsozialisiert ' werden kann, sondern ist essenziell auf ständige symbolische Verdeutlichung angewiesen. Ihre Überlebens- und Anpassungsfähigkeit, ihr Reichtum und ihre Qualität hängen stets auch davon ab, ob neue Denk- und Handlungsmöglichkeiten erschlossen und/oder in Vergessenheit geratene Lebensweisen wieder in Erinnerung gerufen werden, ob neue Symbole gestiftet, und/oder vorhandene Sinnbezüge wieder sinnenfällig gemacht werden“ (ebd.: S. 42f.). Im Kontext dieses Verständnisses sowie im Zuge der Entwicklung von Mediengesellschaften (Donges und Jarren 2017) hat sowohl die politische Kommunikationsforschung insgesamt (Sarcinelli 1989, 2011) als auch die visuelle Kommunikationsforschung im Speziellen an Bedeutung gewonnen (Aiello und Parry 2020; Müller und Geise 2015; Veneti et al. 2019). Maßgebliche Impulse für ikonische Ansätze in der deutschsprachigen Politikwissenschaft gingen vom Arbeitskreis Film und Politik/Visuelle Politik der Deutschen Vereinigung für Politikwissenschaft ${ }^{2}$ aus (Hofmann 1999a; vgl. auch Drechsel 2009 sowie Münkler und Hacke 2009).

So kritisierte Marion Müller die Vernachlässigung der visuellen Dimension von Politik mit dem Verweis auf vorherrschende diskursive Öffentlichkeitsbegriffe, die Öffentlichkeit ausschließlich unter dem Aspekt rationaler Funktionalität konzeptualisieren (Müller 1999, S. 18). Mit Blick auf eine von Massenmedien vermittelte politische Wirklichkeit, könne Öffentlichkeit jedoch eine über argumentative Strukturen hinausweisende Dimension zugeschrieben werden. In einer ,[i]konische[n] Öffentlichkeit“" (ebd.: S. 19) kommen der bildzentrierten und der wortzentrierten Kommunikation gleichwertige Rollen zu. Die beiden Kommunikationsformen sind als überlappend zu begreifen, unterscheiden sich jedoch hinsichtlich ihres Funktionsprinzips. Im Gegensatz zur letzteren folgt erstere einer assoziativen Logik, die nicht rational erklärbar ist, auf Vorbildern beruht und eine deutliche emotionale Komponente aufweist. Insofern wird Müllers Konzept der politischen Vision, hier verstanden als Synonym für das Bildhafte der Politik, als nützlich angesehen, um eine stark an Output-Leistungen orientierte Politikwissenschaft durch Fragen nach dem Input in Form von ,Wahrnehmungs- und Kommunikationsstrukturen von Politik“ (ebd.: S. 20) zu ergänzen.

Einen ähnlichen Ansatz verfolgt Wilhelm Hofmann (1999b), der eine Theorie der visuellen Politik im Zusammenhang mit Fragen nach der (Un-)Sichtbarkeit von Macht und Herrschaft auch als eine Theorie der Demokratie (Hofmann 2009, S. 112) versteht. Politik, so die verbindende These dieser Konzepte, wird immer auch über visuelle Symbole vermittelt und zwar unabhängig davon, ob die individuelle Ebene, die Kommunikation zwischen Organisationen auf einer Meso-Ebene oder die Ver-

\footnotetext{
2 Der Arbeitskreis firmiert heute unter dem Namen Politik und Kultur.
} 
mittlung von Politik durch Massenmedien im Fokus steht (Drechsel 2009, S. 161). Zudem plädieren diese Ansätze dafür, die Erforschung der Zeichenwelten interdisziplinär auszurichten und dabei kunst-, sozial- und kommunikationswissenschaftliche Theorien und Methoden miteinander zu verknüpfen (Kappas und Müller 2006; Veneti et al. 2019).

Wie Giorgia Aiello und Katy Parry (2020) konstatieren, ist die oben geschilderte Dichotomie zwischen einer argumentativ-rationalen und einer assoziativ-emotionalen Dimension von Kommunikation traditionell ebenso umstritten wie die normative Frage, ob visuelle Medien ,,besser“ oder „,schlechter“ für den öffentlichen Diskurs sind. Davon ausgehend, dass Bilder gleichermaßen Informations- wie Manipulationszwecken dienen können, ist es jedoch geboten, achtsam für die vielfältigen Erscheinungsformen von visuellen Medien in der öffentlichen Kommunikation zu sein (110 ff.). ${ }^{3}$ Wie Forschungsergebnisse aus der Medienpsychologie zeigen, beeinflusst zum Beispiel die Art der visuellen Darstellung eines skandalisierten Politikers (isoliert oder zusammen mit anderen Politiker*innen) die Wahrnehmung der Rezipient*innen (von Sikorski und Ludwig 2018). Doch nicht nur Experimente verdeutlichen die Möglichkeiten, wie durch visuelle Medien Realitäten geschaffen werden. Mittels Rekonstruktionen von Pressefotografien in Tageszeitungen lassen sich die vielfältigen unterschiedlichen Auswahlen, Zuschnitte und Modulationen von Bildern und die damit verbundene ,,ikonische Macht“ (Kanter 2016) von Redaktionen aufzeigen. Neurophysiologische Studien legen nahe, dass derartige redaktionelle Routinen eng an bestimmte Prinzipien in der ästhetischen Erfahrung des Menschen gekoppelt sind. Die Gruppierung von Objekten in einem Bild, die Orientierung an Symmetrien und die Verwendung von Kontrasten nutzen Künstler*innen und Medienschaffende, um bestimmte Effekte zu erzielen (Ramachandran und Hirstein 1999).

\subsection{Abbilder und Denkbilder}

Wenngleich bestimmte Prozesse durch das visuelle System festgelegt zu sein scheinen, ist die Bedeutungszuschreibung von Bildern in hohem Maße kontextabhängig, da sie auf Vorerfahrungen beruht, die wiederum durch zeitliche, kulturelle, soziale sowie individuelle Wahrnehmungsdifferenzen bedingt sind. Visuelle Medien haben demzufolge einen stark polysemen Charakter (Geise und Baden 2015, S. 52). Diese Ambiguitäten in der Wahrnehmung lassen sich mit Aby Warburgs Unterscheidung zwischen Abbildern (materiellen Bildern) und subjektiv unterschiedlichen Denkbildern (mentalen Bildern) verdeutlichen. Dabei wird angenommen, dass jedes Abbild ein Denkbild produziert, aber ein Denkbild nicht zwangsläufig ein Abbild (Müller 2011, S. 46). Das Abbild eines Apfels beispielsweise kann mit dem Denkbild gesunder Ernährung, dem Sündenfall oder einem bekannten Technologieunternehmen korrespondieren.

Diese dualistische Konzeption von Ab- und Denkbild lässt sich für die Zwecke sozialwissenschaftlicher Analyse in ein breit angelegtes Bildkontextmodell einbetten, das zwischen den drei Dimensionen Form, Produktionskontext und Wirkungskontext differenziert. Müller setzt diese drei Kontextstrata in Analogie zum Dreistufenmodell

\footnotetext{
3 Zur Problematisierung der Manipulation vgl. Drechsel (2009, S. 150f.) und Hofmann (2009, S. 114f.).
} 
des Kunsthistorikers Erwin Panofsky, das aus der vor-ikonographischen Beschreibung, der ikonographischen Analyse und der ikonologischen Interpretation besteht (ebd.: S. 46; Panofsky 1975). Angewendet auf die vorliegende Untersuchung heißt das: Bei den Covern handelt es sich formal betrachtet um Bilder in einem journalistischen Produktionskontext. Sie entstehen in der Redaktion des Magazins bzw. werden von dieser in Auftrag gegeben. Der Rezeptionskontext bleibt hier auf die Betrachtung und Interpretation des Forschers beschränkt. Bisweilen wird kritisiert, dass diese Interpretationen nichts über die allgemeine Rezeptionsweise aussagen (Gerth 2012, S. 204). Das mag stimmen, ist jedoch nicht problematisch, da verallgemeinerbare Aussagen zur Medienrezeption grundsätzlich nicht im Erkenntnisinteresse ikonographisch-ikonologischer Forschung liegen. Vielmehr steht die Rekonstruktion möglicher Bildbedeutungen im Mittelpunkt. Das Vorgehen ist geprägt durch ein stetiges Eintauchen ins Material, das die ,informierten Vorannahmen“" (Yanow 2014, S. 101) schärft und dadurch im Sinne eines iterativen Prozesses zum Erkenntnisgewinn beiträgt.

\subsection{Visuelles Framing}

Ausgehend von einer Perspektive, die Massenmedien einen realitätskonstruierenden Charakter zuschreibt, sucht dieser Beitrag nach visuellen Medienframes (Geise et al. 2013; Müller und Geise 2015, S. 269f.), welche die Bildberichterstattung über die NPD und die AfD in ihren Aufstiegs- bzw. Etablierungsphasen leiten. Der auf Erving Goffman (1974) zurückgehende Frame-Ansatz findet seit Anfang der 1990er Jahre vermehrt in der Auseinandersetzung mit massenmedial verbreiteten Informationen Anwendung (Dahinden 2006; Marcinkowski 2014) und hat sich neben Agenda Setting und Priming als eines der Schlüsselkonzepte in der politischen Kommunikationsforschung etabliert. Während sich Agenda Setting laut Holli Semetko (2007, S. $131 \mathrm{ff}$.) auf die Auswahl von Themen bezieht, die medial Beachtung finden, und Priming beschreibt, welche Aspekte entscheidend für die Informationsverarbeitung, Meinungsbildung und Bewertung politischer Sachverhalte, Politiker*innen und Parteien sind, fokussiert der Framing-Ansatz die Art und Weise, wie bestimmte Themen medial dargestellt bzw. wahrgenommen werden.

Inzwischen existiert ein beträchtlicher Literaturkanon zu Framing und Frames. Auf theoretischer Ebene liefert Matthias Potthoff (2012) eine Systematisierung, in der er die Ansätze aus verschiedenen Disziplinen synthetisiert. Die methodische Bandbreite aus kommunikationswissenschaftlicher Sicht zeigt Jörg Matthes (2008) in einer Metastudie auf. Framing - darüber herrscht weitgehend Konsens - wird zunächst als dynamischer Prozess begriffen, der auf verschiedenen Ebenen (Kommunikator*innen, Medienschaffende und Rezipient*innen) angesiedelt ist, die sich gegenseitig beeinflussen. Frames sind die Ergebnisse dieses Prozesses. Sie sind nicht als statisch zu betrachten, sondern sind über die Zeit veränderlich (Entman et al. 2009, S. 177 ff.). Frames stellen Deutungsmuster dar (Dahinden 2006, S. 308), die bestimmte Sichtweisen und Kontextualisierungen eines Themas sichtbar machen. Die Frame-Analyse untersucht dementsprechend, the selection and salience

\footnotetext{
${ }^{4}$ Eigene Übersetzung.
} 
of certain aspects of an issue by exploring images, stereotypes, metaphors, actors and messages“" (Entman et al. 2009, S. 180).

Verknüpft man nun die Überlegungen zur visuellen Politik mit der Beobachtung, dass sich die Frage der politischen Kommunikation populistischer Parteien im Rahmen heuristischer Mikro-Makro-Kommunikationsmodelle zu einem zentralen Thema für die Forschung entwickelt hat (Aalberg et al. 2017; Herkman 2016), ist es gewinnbringend herauszuarbeiten, wie Medien das Erstarken der AfD visuell rahmen. Die Untersuchung, inwiefern vergleichbare Deutungsmuster in der Visualisierung des Aufstiegs der NPD in den 1960er Jahren vorliegen, kann Aufschluss darüber geben, ob Der Spiegel in der Gegenwart im Sinne von Paul (2008) auf einen bekannten Bilderkanon zurückgreift und damit an in der Vergangenheit genutzte Narrative anknüpft. In Bezug auf die „Sichtbarkeit der Macht“ (Hofmann 1999a) lässt sich ferner aufdecken, welche Elemente politischer Macht medial sichtbar gemacht werden und welche nicht.

\section{Methodik}

\subsection{Magazincover als Datenmaterial}

Magazine sind ,windows on cultural conditions in any given time and place“ (Kitch 2018, S. 14). Sie bringen die ihrer Leserschaft unterstellten Vorstellungen und Wünsche möglichst weitgehend zum Ausdruck ${ }^{5}$ und tendieren dazu, Bilder des in einer Gesellschaft jeweils aktuellen Bilderkanons zu reproduzieren. Damit tragen sie wiederum zu deren weiteren Verankerung im kulturellen Gedächtnis bei (Paul 2008, S. 33). Den Titelbildern kommt dabei in mehrfacher Hinsicht eine besondere Rolle zu. Sie sind nicht nur Indikator dessen, was aktuell diskussionswürdig erscheint, sondern fungieren auch als Blickfänger und werbende Aushängeschilder (Spiker 2018, S. 377). Sie sollen Aufmerksamkeit erregen und zum Kauf animieren.

Die Titelbilder des Spiegels können als in hohem Maße artifiziell und anspielungsreich gelten (Schütt und Schwarzkopf 1999), was sie für eine politikwissenschaftliche Analyse unter bildwissenschaftlichen Vorzeichen qualifiziert. Insofern werden dessen Cover hier als ,historische Quellen verstanden, durch deren ikonografisch-ikonologische Analyse der gesamte soziopolitisch-kulturelle Entstehungskontext beleuchtet werden kann und umgekehrt die Bedeutungen des Bildmaterials anhand dieser Kontextrekonstruktionen erschlossen werden können“ (Müller 2011, S. 29). Die politisch-kulturelle Bedeutung des Spiegels wird dadurch verstärkt, dass er nach wie vor als auflagenstärkstes deutsches Nachrichtenmagazin gelistet ist. In den ersten Quartalen der Jahrgänge 1966 und 2016 wurden rund 686.000 bzw. 793.000 Exemplare pro Ausgabe verkauft (IVW 2019). Da das Einzelheft in der Regel von mehreren Personen gelesen wird, geht die Reichweite weit über diese

\footnotetext{
5 Der Spiegel ist politisch tendenziell linksorientiert, weshalb eine kritische Distanz zu politisch rechtsorientierten Parteien zu erwarten ist. Das eher bürgerlich konservativ ausgerichtete ebenfalls wöchentlich erscheinende Magazin Focus kommt als mögliches Vergleichsmedium für diese Zeitspanne nicht in Frage, da es erst seit 1993 erscheint.
} 
Zahlen hinaus, sodass das Magazin über ein erhebliches Potenzial für die öffentliche Meinungsbildung verfügt.

\subsection{Untersuchungszeiträume und Fallauswahl}

Ausschlaggebend für die Hinzunahme von Covern zur NPD Mitte der 1960er Jahre ist die Parallelität des bundesweiten Erstarkens einer Partei rechts der Union. Nach dem Verbot der Sozialistischen Reichspartei (SRP), war die NPD die erste überregional erfolgreiche Partei rechts der CDU und erzielte 1966 Erfolge bei Landtagswahlen in Hessen und Bayern. Mit der AfD erscheint 2013 erneut eine Partei rechts der Union. Sie ist derzeit flächendeckend in den Landesparlamenten und seit $2017 \mathrm{im}$ Bundestag vertreten. Da weitere potenziell relevante Akteure wie die 1971 gegründete Bewegung Deutsche Volksunion (DVU), die im Zuge der Debatte um den Milliardenkredit an die DDR 1983 gegründete Partei Die Republikaner (REP) sowie die pro-Bewegung im Vergleich zur NPD und zur AfD lediglich regional begrenzte Erfolge erzielten (Jaschke 2016; Pfahl-Traughber 2019, S. $71 \mathrm{ff}$.) und somit von einer geringen Bedeutung für die überregionale Presse ausgegangen werden kann, bleiben diese hier unbeachtet.

Die Untersuchungszeiträume lassen sich anhand des sogenannten media life cycle Modells bestimmen. Es unterteilt die Beziehung zwischen Massenmedien und populistischen Parteien idealtypisch in vier Phasen: ,ground-laying phase“, ,insurgent phase“, ,established phase“, ,decline phase“ (Mazzoleni 2008, S. 59 ff.). Die Gründungsdaten der Parteien bilden den jeweiligen Beginn der Untersuchungszeiträume: für die NPD ist dies der 28. November 1964, für die AfD der 6. Februar 2013. Als Endpunkt (decline phase) wird für die NPD der knapp verpasste Einzug in den Bundestag 1969 festgesetzt. Die AfD befindet sich aktuell in der etablierten Phase, sodass der Untersuchungszeitraum bis Dezember 2019 reicht.

\subsection{Die ikonographisch-ikonologische Analyse}

Für die Einzelbildanalyse wird das von Marotzki und Stoetzer (2006) entwickelte vier Phasen umfassende Verfahren genutzt, das sich an den ikonologischen Studien Panofskys (1975) orientiert. Die im Folgenden dargelegte kleinschrittige Vorgehensweise erlaubt eine explorative Analyse, die nah am Datenmaterial erfolgt und damit eine hohe Nachvollziehbarkeit gewährleistet. Sie eignet sich, um die Bandbreite an möglichen Bedeutungen der Bildobjekte in ihrer Art und Weise der Darstellung sowie ihrem Verwendungskontext offenzulegen. Während Ikonographie, dem Suffix -graphie entsprechend, die Beschreibung einzelner Elemente meint, bezeichnet Ikonologie (abgeleitet von logos: Denken, Vernunft) eine über das Deskriptive hinausgehende Interpretation (ebd.: S. 42).

Der erste Schritt bewegt sich auf der Objektebene und beinhaltet eine Beschreibung der Bildobjekte. Dieser aus Platzgründen häufig nicht verschriftlichte Arbeitsschritt findet hier explizit Erwähnung, da die Beschreibung des Visuellen das ursprünglich Bildhafte erst in den folgenden Schritten einer ikonologischen Interpretation zugänglich macht (Pilarczyk 2017, S. 83). 
Im zweiten Schritt, der Ordnung der Objekte, liegt der Fokus auf der Bedeutung der Objekte sowie ihrer Einordnung in einen Sinnzusammenhang, der sich aus dem jeweiligen Kulturkontext erschließt, in dem das Bild entstanden ist (vgl. Panofsky 1975, S. 39). Die Konstruktion des Sinnzusammenhangs führt zu einer Narration, d.h. Bildmotive und Bildthema werden mit einer Geschichte in Verbindung gebracht (Marotzki und Stoetzer 2006, S. 22).

Anschließend wird drittens die Inszenierung (mise-en-scène) untersucht. Während sich die ersten Schritte mit der Frage befassen, was abgebildet ist, wird in diesem Schritt das Augenmerk auf das Wie gelegt. Hier sind gestalterische Aspekte wie das Zusammenspiel von Farben, die Perspektive der Aufnahme sowie Vorder- und Hintergrund entscheidend. Diese sind insofern relevant, als sich klassische Künstler*innen wie auch moderne Illustrator*innen bei der Bildgestaltung an „,Gesetzmäßigkeiten“ (Ramachandran und Hirstein 1999, S. 17) orientieren, die für die visuelle Wahrnehmung maßgeblich sind.

In der abschließenden Synthese wird der Zusammenhang zwischen Bildinhalt und gesellschaftlichem Gehalt des Bildes herausgearbeitet. Externe Quellen ${ }^{6}$ und das Hintergrundwissen des Forschers zur politischen Lage werden mit den Ergebnissen der vorausgehenden Analyseschritte verknüpft und eine ikonologische Interpretation formuliert.

\section{Empirische Analyse}

Das erstmalige Erscheinen der jeweiligen Partei auf einem Titelbild wurde als Kriterium für eine detaillierte Analyse festgelegt. Somit werden zunächst die Cover 1966, Nr. 15 und 2016, Nr. 6 ausführlich untersucht. Um den Analyserahmen auszuweiten und mögliche weitere visuelle Deutungsmuster erfassen zu können, werden weitere vier Cover aus dem angegebenen Untersuchungszeiträumen vorgestellt, welche die NPD bzw. die AfD in visueller Form darstellen und die Bedeutung der Parteien für die innerdeutschen Machtverhältnisse thematisieren.

\subsection{Auf- oder Untergang der Geschichte? (Abb. 1)}

Objektebene: In der Bildmitte des Covers sind ein großer weißer Kreis und darin ein blauer Schriftzug zu sehen. Der Kreis ist von roter Farbe umgeben. Das untere Viertel des Bildes ist schwarz und eine leicht unregelmäßige horizontale Linie zieht sich über den untersten Teil des Kreises. Auf der schwarzen Fläche steht ein weißer Schriftzug und darüber ragt ein schwarzes kreuzförmiges Zeichen in den Kreis hinein. Zum Teil überdeckt dieses die blauen Schriftzeichen.

Ordnung der Objekte: Bei dem blauen Schriftzug handelt es sich um die Abkürzung der Nationaldemokratischen Partei Deutschlands (NPD). Das schwarze Zeichen darunter stellt ein Hakenkreuz dar, das hier als Symbol der Nationalsozialistischen Deutschen Arbeiterpartei (NSDAP) bzw. als offizielle Reichs- und Nationalflagge

\footnotetext{
${ }^{6}$ In Anlehnung an Gérard Genette (2001) gehen sowohl Peritexte, wie der zum Titelbild gehörige Artikel, als auch Epitexte in Form zeitgenössischer wissenschaftlicher Analysen in die Synthese mit ein.
} 
Abb. 1 Der Spiegel vom 4. April 1966, Nr. 15

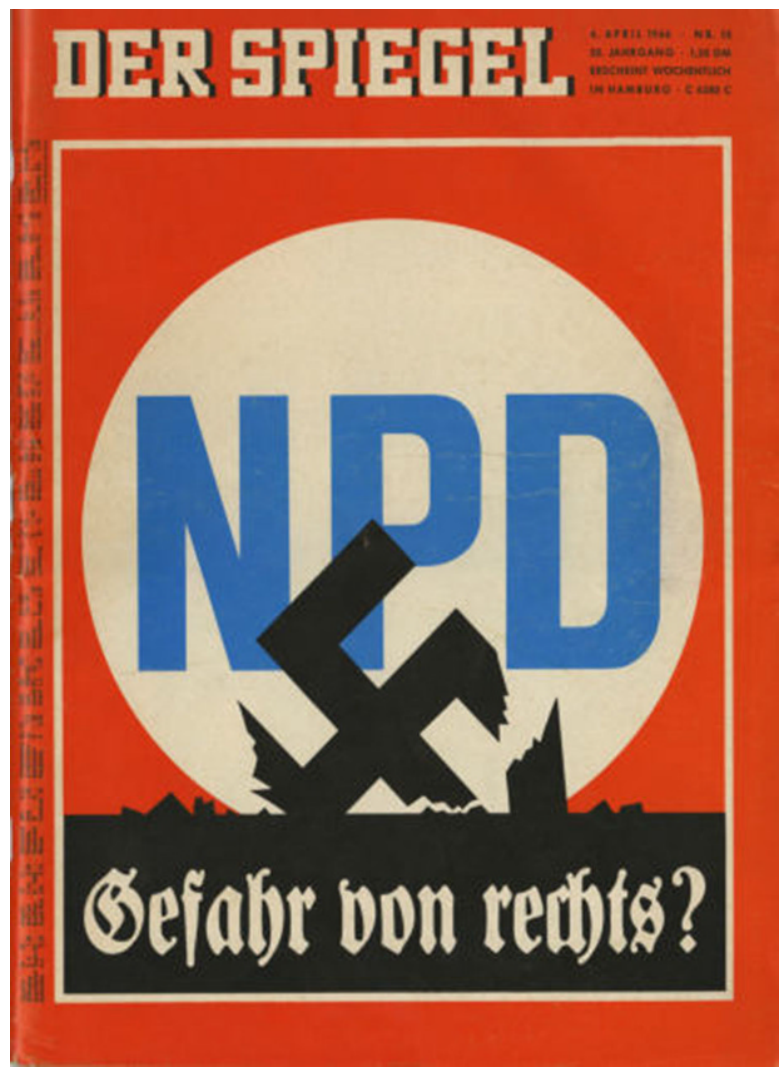

des NS-Staats zu verstehen ist. An den Haken sind teilweise Brüche sichtbar, was sinnbildlich als Zerstörung und folglich als Ende des Nationalsozialismus aufgefasst werden kann. Zugleich verweisen die Trümmer auf die Zerstörung durch den Krieg. Der rote Hintergrund bildet nicht nur die Farbe der Logos von NSDAP und NPD ab, sondern lässt sich auch als Warnsignal vor der im Titel genannten Gefahr deuten.

Mise-en-scène: Im Kontext des NS-Bezugs erinnert die Verschmelzung der verschiedenen Bildobjekte an die Collagen des dadaistischen Künstlers John Heartfield, in denen er den Aufstieg der NSDAP thematisierte (Coles 2014). Durch die Darstellung des weißen Kreises, der hinter dem schwarzen Vordergrund erscheint, entsteht der Eindruck eines Sonnenaufgangs. Einerseits wirkt die Darstellung so, als sei das Hakenkreuz aus der Mitte des Kreises herausgefallen, zerbrochen und der Nationalsozialismus somit untergegangen. Andererseits steigt das offizielle NPD-Logo hinter dem Hakenkreuz auf, wodurch der Eindruck entsteht, dass die Partei die Ideologie der NSDAP in neuem Gewand hervorbringt. Insofern erzeugt die vertikale Inszenierung eine Dynamik, die auf einen Übergang von unten nach oben verweist. Die Art der Darstellung impliziert eine lineare Entwicklung von der NSDAP hin zur NPD. Dieser vertikale Aufbau lässt sich als diachrone Argumentation deuten, welche die Frage nach einer drohenden „Gefahr von rechts“ implizit mit „Ja“ beantwortet. Der 
Bezug zur rechten Gefahr wird überdies durch die von den Nationalsozialisten instrumentalisierte Frakturschrift hergestellt. ${ }^{7}$ Insofern tragen hier auch typographische Elemente durch entsprechendes Layout zur Konstituierung eines visuellen Frames bei (Rössler 2014, S. 47).

Versteht man die schwarze Fläche nicht historisch im Sinne der Kriegstrümmer aus der Vergangenheit, sondern räumlich, eröffnet sich die Deutungsmöglichkeit nicht näher spezifizierter Gruppen in der Bevölkerung, die als Fundament für die Verbreitung rechtsextremen Gedankenguts fungieren. In der Tat spielt diese Lesart in der zeitgenössischen Debatte zur NPD eine tragende Rolle, wie die nachfolgende Synthese zeigt.

Synthese: Das Titelbild erschien im April 1966. Bei der Bundestagswahl im Herbst des Vorjahres kam die NPD zwar lediglich auf 2,0\% der Stimmen. Die Befürchtung, dass sie bei den anstehenden Landtagswahlen in Hessen und Bayern besser abschneiden würde, war in weiten Teilen der Bevölkerung jedoch groß. Schließlich zog die NPD im November 1966 mit 7,9\% und 7,4\% in den hessischen und den bayerischen Landtag ein. Besonderer Anlass zur Sorge für viele war die Tatsache, dass der Gründungsvorstand der NPD zu großen Teilen aus ehemaligen aktiven NSDAP-Mitgliedern bestand (Niethammer 1969, S. 277 ff.). ${ }^{8}$ Sowohl die personelle Aufstellung als auch die programmatische Ausrichtung der NPD zwischen konservativen und radikalen Kräften wurde in der Öffentlichkeit heftig diskutiert (Der Spiegel 1966, S. 33).

Der zeitgenössische wissenschaftliche Diskurs lässt sich grob in zwei Stoßrichtungen unterteilen. Auf der einen Seite stehen kritische Auseinandersetzungen mit der NPD, die sich überwiegend mit faschistischen und neonazistischen Elementen der Partei beschäftigten (Kühnl 1967; Niethammer 1969). Auf der anderen Seite sind Analysen zu finden, die politisch-kulturelle Charakteristika in den Blick nehmen und grundsätzlich den Grad der Konsolidierung der bundesrepublikanischen Demokratie bzw. ihrer Verfassung zur Zeit der Großen Koalition diskutieren (u.a. Dahrendorf 1968, S. 92 ff.). Iring Fetscher identifiziert zu dieser Zeit ein zunächst

\footnotetext{
${ }^{7}$ Diese Schriftart war keine Erfindung der nationalsozialistischen Führung. Sie blickt auf eine jahrhundertealte Tradition zurück (Keunecke 1993, S. B121). Der Reichsinnenminister Wilhelm Frick und der „Bund für deutsche Schrift" trugen ab 1933 jedoch maßgeblich dazu bei, dass die Frakturschrift gegenüber der lateinischen (Antiqua) eine vorrangige Stellung einnahm. Als Zeichen der Abgrenzung ordnete das Propagandaministerium 1937 an, dass jüdische Verlage fortan die Antiqua zu nutzen hätten. Im sogenannten Normalschrifterlass 1941 wurde die Fraktur dann überraschend verboten und die Antiqua im Gegenzug als „Normalschrift“ deklariert. Zum einen, so die Argumentation, hätten Juden die Schrift für sich vereinnahmt [,Schwabacher-Judenlettern“ (zit. nach ebd.: B123)]. Zum anderen war die Frakturschrift den Bevölkerungen in den besetzten Gebieten nicht geläufig, was die Kommunikation erschwerte. Heute findet die Fraktur u. a. in Logos neonazistischer Bands und Modelabels Verwendung (Miller-Idriss 2017, S. 61). Eine Gleichsetzung der Fraktur als Nazi-Schrift erscheint jedoch nicht nur aus den genannten historischen Gründen problematisch. Deutlich wird die Kontextabhängigkeit für die Bedeutung der Schriftart an weiteren Beispielen. So ist die Fraktur in der Gestaltung von Zeitungsköpfen verbreitet oder in der Werbung, wo sie ein Bild von Tradition und Qualität zu vermitteln versucht.

8 Wenngleich das nicht ausschließlich für die NPD galt: „Alte Nazis gibt es nicht nur in der NPD und nicht nur Demokraten wählen die etablierten Parteien. Die NPD ist nur ein Teil der deutschen Rechten, die auch in CDU, CSU, FDP und SPD siedelt. Daß sie allein - zumal im Ausland - Furcht erweckt, erklärt sich daraus, daß sie offen am Revers trägt, was in der Brust der anderen schlummert“" (Der Spiegel 1968, S. 31).
} 
von der NPD unabhängiges antidemokratisches und antiliberales Potenzial in der bürgerlichen Mitte und verbindet dies mit einer Kritik an den Volksparteien und Publizist*innen sozialdemokratischer wie konservativer Prägung. Diese hätten Topoi verbreitet, die rechtsradikalen Kräften Nährboden lieferten und sie somit erst stark machten: so beispielsweise die Ablehnung vieler Politiker*innen, Feierstunden zum 20. Jahrestag der Befreiung vom Nationalsozialismus abzuhalten sowie die angeblich diskriminierende Behandlung deutscher Kriegsverbrecher oder die von Ludwig Erhard vorgetragenen Worte vom Ende der Nachkriegszeit (Fetscher 1967, S. 16).

Eine ähnliche Perspektive prägt die vielzitierte Analyse von Peter Dudek und Hans-Gerd Jaschke (1984). Ein Großteil der Anschauungen der NPD sei nicht nur von der Union in gleichem Maße vertreten, sondern von weiten Teilen der Bevölkerung mitgetragen worden. Folglich sei ,[d]ie Etikettierung der NPD als extremistisch oder neonazistisch [...] daher immer auch das Verdrängen der Einsicht, daß [...] viele der an der NPD kritisierten Politikstrategien zugleich auch anerkannte und wirksame Fermente der politischen Kultur der Bundesrepublik sind“ (ebd.: S. 350). Die Autoren sehen eine Überbewertung der NS-Symbole und eine Unterbewertung der rechtskonservativen Elemente in der Debatte um die NPD zwischen 1964 und 1969. Dabei handelte es sich um eine Dynamik, die entscheidend von den Massenmedien und hier insbesondere von seriösen - und zwar gleichermaßen von sozialdemokratischen, konservativen und liberalen - Zeitungen unreflektiert verstärkt wurde (ebd. 352). Die NPD hatte damals gegen Der Spiegel, stern und Quick Klage beim Göttinger Landgericht eingereicht. Die Partei warf den drei Magazinen Verleumdung vor, da sie behaupteten, die NPD sei eine Nachfolgeorganisation der NSDAP (Frederik 1966, S. 54). Die Klagen wurden zwar abgewiesen, verdeutlichen aber die Intensität der Debatte, zu der das hier analysierte Cover beigetragen haben dürfte.

Die folgende Analyse zeigt, welche Bedeutung diese Problematik 2016 auch in der Debatte um die AfD hatte.

\subsection{Auf dem Zeppelinfeld (Abb. 2)}

Objektebene: Im Vordergrund erstreckt sich bildmittig das Porträt einer Frau. Ihr Kopf, ihr Hals sowie die Ansätze ihrer Schultern sind zu sehen. Sie trägt kurze dunkle Haare. Ihr Blick ist rechts am Betrachter vorbei in die Ferne gerichtet, wobei ihre Augen zusammengekniffen sind, sodass die Stirn in Falten liegt. Ihr Mund ist geschlossen. Links hinter ihr ist in identischer Pose jedoch deutlich kleiner das Porträt eines älteren Mannes zu erkennen, der in gleicher Richtung in die Ferne schaut. Er trägt formelle Kleidung. Unter den beiden Köpfen steht die Bildunterschrift. Im Hintergrund ist eine Menschenmenge auf einer Tribüne zu erkennen, die oben mit Säulen gesäumt ist. In der Menge sind mehrere Flaggen mit einem Kreuz in der Mitte sowie Flaggen mit drei gleich großen waagrechten Streifen auszumachen. Zudem ist rechts unten eine weitere Flagge mit zwei waagrechten Streifen und einem Zeichen in der Mitte zu sehen.

Ordnung der Objekte: Bei der Frau handelt es sich um die damalige AfD-Bundessprecherin Frauke Petry. Der Mann im Hintergrund ist Alexander Gauland, ebenfalls einflussreicher AfD-Politiker. Im Hintergrund ist die Haupttribüne des Zeppelinfeldes auf dem Reichsparteitagsgelände in Nürnberg abgebildet. Ursprünglich als Wiese 
Abb. 2 Der Spiegel vom 6. Februar 2016, Nr. 6

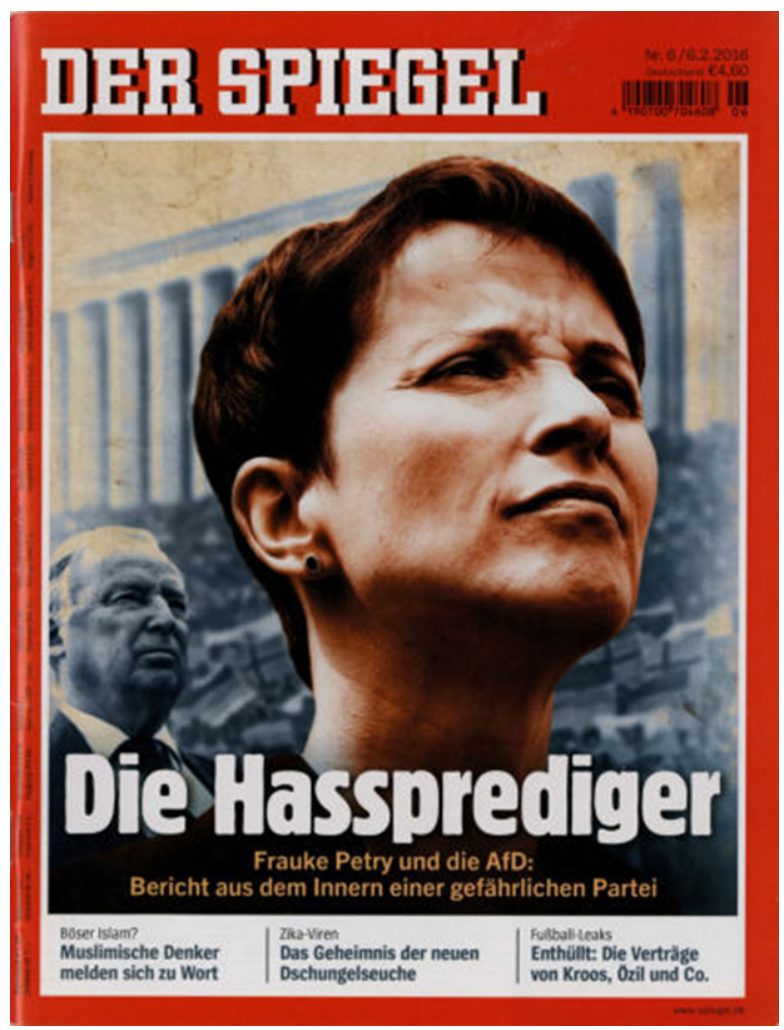

angelegt, ließ Hitler das Feld zwischen 1935 und 1937 zu einer Fläche mit Tribünen umbauen. Die Anlage diente für Aufmärsche und sollte die von der NS-Führung propagierte „Volksgemeinschaft“ erlebbar machen (Steuwer 2017, S. 196ff.). Die Deutschlandflaggen, die sächsische Flagge und die Wirmer-Flaggen ${ }^{9}$ im Bildhintergrund lassen erkennen, dass es sich bei der Menschenmenge auf der Tribüne um PEGIDA-Anhänger*innen handelt.

Mise-en-scène: Die serielle Anordnung Petrys und Gaulands, die Kopfhaltung sowie der durch starke Kontraste verstärkte strenge, militärisch anmutende Gesichtsausdruck erinnern an die Aufmärsche auf dem Zeppelinfeld und an das vom nationalsozialistischen Regime intendierte Aufgehen des Einzelnen im „Volkskörper“. Die schwarz-weiß Fotomontage in Abb. 2 verweist auf den Vergangenheitsbezug und

\footnotetext{
${ }_{9}$ Die Symbolkraft der Wirmer Flagge mit dem golden umrandeten schwarzen Philippuskreuz auf rotem Grund hat einen bemerkenswerten Wandel vollzogen. Sie wurde 1944 von Joseph Wirmer, einem Widerstandskämpfer gegen den Nationalsozialismus entworfen und sollte als Nationalflagge für ein Deutschland nach Hitler eingesetzt werden. Heute dient eine Abwandlung der Flagge als Symbol der German Defence League, einer Organisation, die angibt, die christliche Tradition vor einer drohenden Islamisierung zu schützen. Seit 2015 beanspruchen PEGIDA-Anhänger die Wirmer Flagge zudem als nationalistisches Symbol, wodurch sie zu einem „besondere[n] neo-konservative[n] ,Kampfzeichen““ (Schlürmann 2015, S. 342) geworden ist.
} 
weckt Assoziationen an Szenen aus Leni Riefenstahls Film Triumph des Willens, der die propagandistische Ästhetisierung des Reichsparteitags der NSDAP von 1934 dokumentiert. Hinsichtlich der Inszenierung von politischem Personal steht das Cover in der Tradition von Heartfields Titelbildern für die sozialistische Arbeiter Illustrierte Zeitung (1930-1938), auf denen er Hitler und die NS-Führung in Kombinationen aus Fotomontagen und Überschriften lächerlich machte (Coles 2014, S. 169 ff.).

Vor dem Hintergrund der Frage nach der Verkörperung politischer Kollektivität und der Repräsentation des Volkswillens ist die Inszenierung auf dem Magazincover auch im Zusammenhang mit der ideengeschichtlich vielfach rezipierten Visualisierungsform des Frontispizes des Leviathan von Thomas Hobbes zu sehen. Die vielfältigen Adaptionen dieser Bildtradition durch totalitäre Führungen hat Horst Bredekamp (2020, S. $142 \mathrm{ff}$.) ausführlich dokumentiert. ${ }^{10}$ Zwar füllt das „Volk“ in Abb. 2 - hier in Form vieler einzelner zu einer Masse verschwimmender PEGIDAAnhänger - im Gegensatz zum Leviathan nicht Petrys Rumpf aus, geschweige denn richten die Individuen die Blicke erkennbar auf ihren Kopf. Genau die Deutungen dieser Unterschiede sind jedoch für die totalitären Anleihen an diese Bildtradition und somit auch für den hier inszenierten NS-Vergleich relevant. Die symbolische Differenz zwischen dem Hobbesschen Riesen und den ikonischen Anleihen durch totalitäre Bewegungen im 20. Jahrhundert besteht darin, dass die Figur des Leviathan sowohl den Staat als Institution als auch alle darin vereinten Individuen symbolisiert, wohingegen ,die totalitäre Verkörperung [...] die Idee der Volkssouveränität insofern [verschiebt], als dass sie den Willen des Volkes als denjenigen des Führers gestaltet. [...] Die Volkssouveränität wird vom Führer absorbiert und ist nicht mehr die Souveränität des Volkes, sondern die des Führers“ (Diehl 2018b, S. 29f.). Die Inszenierungen auf dem Reichsparteitagsgelände als öffentlich vermitteltes Erscheinungsbild des „Volks“ spiegeln diese Verkehrung des Repräsentationsprinzips. Im Sinne des Ausrufs „Wir sind das Volk“, der anfänglich bei den Montagsdemonstrationen 1989 in der DDR genutzt wurde und den sich die PEGIDA-Bewegung 2014 zu eigen gemacht hat, wird auf dem Titelbild auf die antipluralistische populistische Denkfigur eines einheitlichen und unmittelbar repräsentierbaren Volkswillens im Sinne von „Wir und nur wir repräsentieren das Volk“ angespielt.

Synthese: Die Veröffentlichung des Covers fällt in eine Phase, in der der einstige thematische Schwerpunkt der AfD, die Kritik an der europäischen Währungspolitik, zumindest in der Kommunikationsstrategie nach außen von der Frage nach dem politischen Umgang mit den Flüchtlingen im Sommer 2015 abgelöst worden war. Das Bild kann als Reaktion auf den von Petry in einem Zeitungsinterview geäußerten Satz, dass die Polizei zur Not ,auch von der Schusswaffe Gebrauch machen“ (zit. nach Der Spiegel 2016, S. 13) müsste, um Flüchtlinge an der Grenze aufzuhalten, gesehen werden. In diesem Zusammenhang ist auch die Betitelung als „Hassprediger“ zu verstehen, die im Diskurs ursprünglich anders besetzt war und überwiegend radikalislamische Prediger bezeichnete. Mit dem Aufkommen der PEGIDA-Bewegung wurde der Begriff jedoch auch auf die dort auftretenden Redner*innen angewendet, die zu Feindlichkeit und Gewalt gegenüber Migrant*innen aufrufen. Insofern

10 Zur Auseinandersetzung mit der Wirkungsgeschichte des Hobbesschen Frontispiz siehe auch Manow et al. (2012). 
verweist die bildliche Darstellung auf die katalysierende Wirkung, die Parteivertreter*innen der AfD auf die PEGIDA-Bewegung ausübten (Vorländer et al. 2018, S. 202).

In der Äußerung Petrys zeigt sich beispielhaft die generelle Strategie der Skandalprovokation mit anschließender Distanzierung seitens der AfD, welche die Ausweitung von Sagbarkeitsfeldern im Diskurs zum Ziel hat. Gaulands Äußerungen über Jérôme Boateng, Björn Höckes Aussage über das Holocaustmahnmal oder die Anfeindungen des AfD-Bundestagsabgeordneten Jens Maier gegen Noah Becker sind weitere Beispiele hierfür (Bey und Wamper 2018, S. $118 \mathrm{f}$, , siehe auch Gadinger und Simon 2019). In Bezug auf die mediale Reaktion dieser Provokation offenbart das Titelbild die systemische Affinität von populistischen Kommunikationsstrategien und massenmedialen Aufmerksamkeitsregeln, deren Funktionsmechanismen sich beide durch Personalisierung, Dramatisierung und Zuspitzung auszeichnen (Diehl 2018a, S. 93). Petrys Andeutung des Schusswaffengebrauchs an der Grenze wird aufgegriffen und bildlich durch einen NS-Vergleich verstärkt. Dabei handelt es sich um eine traditionsreiche Form der Diffamierung des politischen Gegners (Eitz und Stötzel 2009, S. 12), die sowohl im politischen Betrieb zwischen Akteuren unterschiedlicher Parteien als auch medial genutzt wird. Sie löst beim Publikum Empörung aus und generiert weitere Aufmerksamkeit. Die Thematisierung Hitlers als Verkaufsstrategie, die der Historiker Ulrich Herbert - unter anderem mit Bezug auf die zahlreichen Titelgeschichten des Spiegel der letzten Jahrzehnte - einmal mit den Worten „Hitler sells“ (Herbert 2015) beschrieb, lässt sich somit auch auf Hitlerbzw. NS-Vergleiche ausweiten.

Ahistorische mediale Inszenierungen, die wie hier Petry und Gauland mit der Szenerie eines Reichsparteitags in eins setzen und damit eine austauschbare Situation zwischen der NS-Diktatur und heutigen Verhältnissen implizieren, sind jedoch, ähnlich wie in der zeitgenössischen Debatte über die NPD Mitte der 1960er Jahre (s. oben), als problematisch zu erachten: „Mag man auch die Beweggründe teilen, die hinter den vielfältigen Verweisen auf die nationalsozialistische Diktatur stehen, so erweisen sie sich in vielen Fällen als fragwürdig, wenn man sie als Aussagen über die Vergangenheit ernst nimmt" (Steuwer 2017, S. 191). Visuell inszenierte Parallelitäten zwischen rechtspopulistischen bzw. rechtsextremen Parteien und dem Nationalsozialismus gehen somit stets mit der Gefahr einer Verharmlosung der NSDiktatur einher.

\subsection{Parteien im Aufwind (Abb. 3)}

Dass die symbolischen Bezüge zumindest zu nationalistischen Tendenzen aus der deutschen Vergangenheit in der Visualisierung von NPD und AfD keine Einzelfälle sind, zeigen weitere Titelbildanalysen (Abb. 3). Eine 1967 initiierte Serie betitelte Der Spiegel vor den für die NPD schließlich erfolgreichen Landtagswahlen in Rheinland-Pfalz und Niedersachen mit „Der neue Nationalismus“ (Abb. 3a). Vor dem Hintergrund der schwarz-weiß-roten Nationalflagge des Deutschen Reiches ist eine phantasievoll zusammengestellte Uniform zu erkennen, die Assoziationen zum deutschen Nationalismus vom 19. Jahrhundert bis in die Zeit des Nationalsozialismus hinein weckt. Konkrete Deutungen sind nicht möglich, da die Elemente zwar 

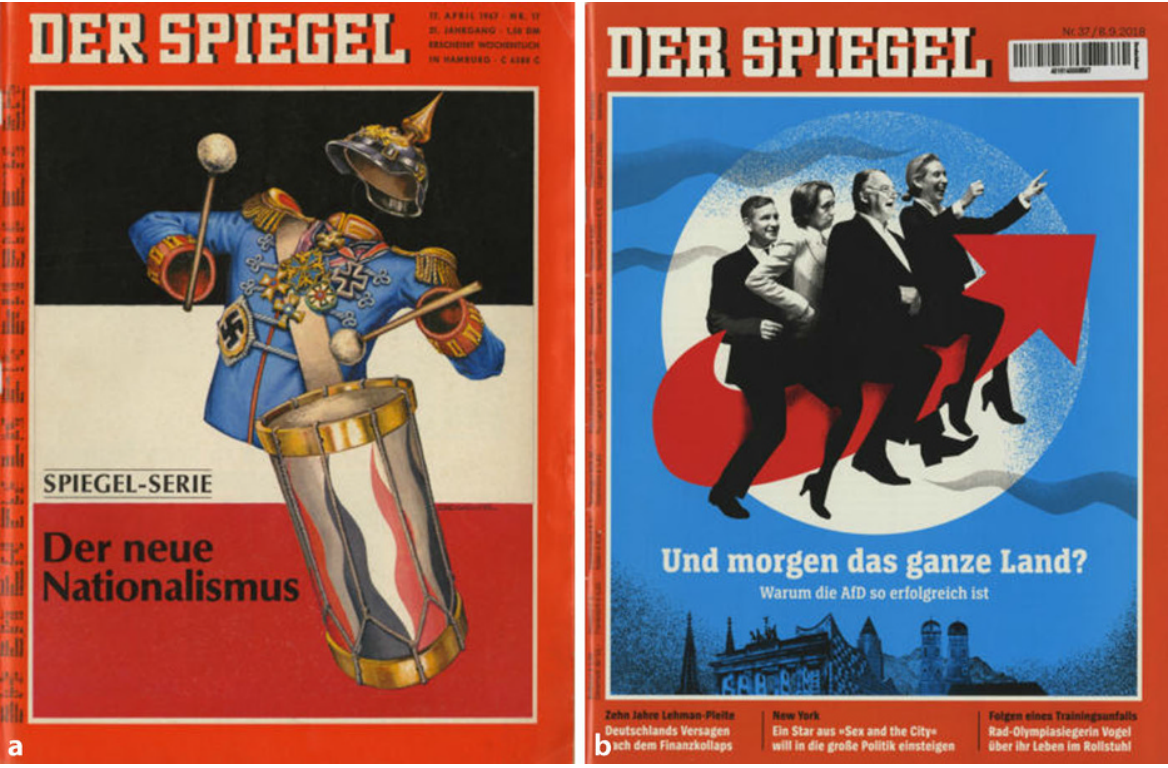

Abb. 3 a Der Spiegel vom 17. April 1967, Nr. 17; b Der Spiegel vom 8. September 2018, Nr. 37

Originalen entlehnt sind, aber nicht exakt in dieser Form gestaltet, geschweige denn in dieser Kombination aus Pickelhaube, Husarenattila, Epauletten und Brustriemen getragen wurden. ${ }^{11}$ Gleichermaßen sind die Orden tatsächlich existierenden Auszeichnungen nachempfunden, in den Details jedoch teilweise der Phantasie des Illustrators entsprungen. ${ }^{12}$ Der Trommler ist ohne Körper und Gesicht abgebildet, was ihn anonymisiert und als prototypische Gestalt des Nationalismus erscheinen lässt. Der Schritt und die Armhaltung deuten an, dass er sich auf dem Vormarsch befindet. Der durch das verwirrende Konglomerat an Abzeichen zunächst nicht definierbare „Geist“ des Nationalismus, kann unter Berücksichtigung des gesellschaftspolitischen Kontexts und der dazugehörigen Titelstory (Der Spiegel 1967) aber als Anspielung auf die NPD verstanden werden. Die Trommel ist nicht nur militärisches Marschinstrument, sondern erinnert an den Roman Die Blechtrommel von Günter Grass (1959), der von der Gesellschaft in Deutschland in der ersten Hälfte des 20. Jahr-

\footnotetext{
${ }^{11}$ Bei dem Helm handelt es sich um eine stilisierte Mannschaftspickelhaube der Infanterie zwischen 1880 und 1914, wobei kein Regiment erkennbar ist und die Kokarde am Riemenende fehlt (Bowman 1989). Der durch die tressenbesetzte Attila angedeutete Husar gehörte einer anderen Truppengattung an, sodass die Uniform militärhistorisch gesehen nicht zum Helm passt. Gleiches gilt für die Ärmelplatten (Funcken und Funcken 1983, S. 122 ff.).

12 Das Kreuz direkt um den Hals stellt einen nicht exakt spezifizierbaren Komturorden dar, der für besondere militärische Verdienste vergeben wurde. Das blaue Kreuz direkt darunter ist der Pour le Mérite, der von Friedrich II. gestiftete höchste Tapferkeitsorden (Fuhrmann 1992). Hinzu kommt das schwarz ausgefüllte Eiserne Kreuz. Es wurde erstmals 1813, erneut 1870 und schließlich 1939, dann von Hitler, gestiftet. Das Cover zeigt letzteres, was am schwarz-weiß-roten Band erkennbar ist (Klietmann 2004, S. 18). Bei dem gestickten aufgenähten Abzeichen unten links handelt es sich um das Deutsche Kreuz in Gold, das ab 1941 eine Zwischenauszeichnung zwischen Eisernem Kreuz und Ritterkreuz darstellte (ebd.: S. 47f.).
} 
hunderts erzählt und die Verführbarkeit der Menschen in der Weimarer Republik und der Zeit des Nationalsozialismus thematisiert.

Die bunte Darstellung des Musikers lässt sich außerdem als Parallele zum Rattenfänger von Hameln ${ }^{13}$ deuten, welcher der Sage nach mit dem Klang seiner Flöte die Ratten aus der Stadt lockte und die Bevölkerung von der Plage befreite. Als ihm der Lohn dafür verwehrt wurde, entführte er, getarnt in neuem Gewand, die Kinder von Hameln und stürzte sie ins Unglück. Diese Interpretationsmöglichkeit schlägt eine Brücke zur politischen Verführungskunst von Demagogen und erinnert an die Folgen des übersteigerten Nationalismus aus dem 19. und 20. Jahrhundert.

In ähnlich spielerischer Form werden nationalistische Tendenzen im Zusammenhang mit der AfD im September 2018 (Abb. 3b) dargestellt. Im Gegensatz zum anonymen „,neuen Nationalismus“ sind hier jedoch explizit Vertreter*innen der Partei abgebildet. Björn Höcke, Beatrix von Storch, Alexander Gauland und Alice Weidel (v.l.n.r.) werden auf dem Pfeil des AfD-Logos über deutschen Städten schwebend als Hexen und Magier inszeniert. Ähnlich der Marschbewegung des personifizierten und zugleich anonymisierten Nationalismus in Abb. 3a wird auch hier eine Dynamik angedeutet. So kann der Hexenritt mit einem nach oben deutenden Pfeil als Besen sinnbildlich für eine im Aufwind befindliche AfD gesehen werden. Das Bild der Hexe erinnert ferner an eine mystische Macht, die sich den Spielregeln der Rationalität entzieht. Insofern verweist die Darstellung auf die von der AfD beförderte Emotionalisierung und die damit einhergehende Irrationalität im öffentlichen Diskurs, die auf Kosten rationaler Argumente an Bedeutung gewinnt (Gadinger und Simon 2019; Korte 2015).

Der Titel „Und morgen das ganze Land?“ ist der umstrittenen Zeile „und heute gehört uns Deutschland - und morgen die ganze Welt"14 aus dem Lied Es zittern die morschen Knochen von Hans Baumann entlehnt, das in den Organisationen des Nationalsozialismus gesungen wurde. Mit „das ganze Land“ ist in Abb. 3b der im September $2017 \mathrm{zu}$ erwartende Einzug der AfD in den bayerischen und den hessischen Landtag gemeint, mit dem die Partei „morgen“ in allen Landesparlamenten sowie im Bundestag vertreten sein würde. Der Mond und die angedeutete Dämmerung symbolisieren einerseits den Vorabend der Wahlen und erzeugen eine mystische Atmosphäre, die mit der Assoziation der Hexen in Einklang steht. Andererseits bildet der angedeutete Vollmond durch die Kreisform eine Parallele zum NPD-Logo und der Hakenkreuzflagge, wenngleich das AfD-Logo in Wirklichkeit keinen weißen Kreis beinhaltet. Somit wird erneut ein Bezug zur nationalistischen und nationalsozialistischen Vergangenheit hergestellt, der im Zusammenhang mit dem Titel an Eroberungsabsichten erinnert.

\footnotetext{
13 Für einen Überblick über verschiedene Versionen dieser Geschichte sowie Karikaturen des Motivs vgl. Humburg (1990).

14 In einer anderen Fassung heißt es „heute da hört uns Deutschland und morgen die ganze Welt“. Winfried Mogge liefert eine Untersuchung zum Ursprung des Lieds, zu den verschiedenen Textvarianten sowie zur juristischen Kontroverse im Nürnberger Prozess gegen die Hauptkriegsverbrecher. Er kommt zu dem Schluss: „Die Differenzierung, ob und wann es ,heute gehört“ oder ,heute da hört uns Deutschland“ hieß, erweist sich als nebensächlich. Der Text, gesteigert durch eingängige Melodie und suggestiven Marschrhythmus, bietet so oder so in den einzelnen Formulierungen und im gesamten Inhalt verbal und mental ein großes Potential an Gewalt- und Zerstörungsbereitschaft“ (Mogge 2007, S. 183).
} 


\subsection{Nach den Wahlen (Abb. 4)}

Abschließend seien zwei weitere Cover vorgestellt, die Parteivertreter*innen kurz nach entscheidenden Wahlerfolgen zeigen (Abb. 4). Das Titelbild in Abb. 4a erschien Ende November 1966, nachdem feststand, dass die NPD erstmals in den hessischen und den bayerischen Landtag einziehen würde. Es zeigt den damaligen NPD-Parteivorsitzenden Friedrich Thielen (r.) und dessen Stellvertreter Wilhelm Gutmann. Das Bild stammt jedoch vom vorangegangenen NPD-Parteitag im Juni 1966 in Karlsruhe. Trotz des seriösen Settings ähnelt die Art der Darstellung den Prototypen von sogenannten Apparatschiks, jenen belächelten Funktionär*innen, die ,menschgewordene Apparat[e]" (Bourdieu 1997, S. 44f.) verkörpern und ihre gesellschaftliche Funktion einzig dem bürokratischen Apparat verdanken. Wäre das im Verhältnis übergroße NPD-Logo vor dem Rednerpult nicht zu sehen, könnte man meinen, es handele sich um eine stereotype Abbildung von Kaderangehörigen. Lediglich das Parteilogo und die Unterschrift „Ruck nach rechts“ lassen einen konkreten Parteibezug erkennen.

Während für die Visualisierung der NPD-Politiker in diesem Beispiel eine nüchtern wirkende Fotografie genutzt wurde, zeigt das Titelbild der Sonderausgabe nach der Bundestagswahl von 2017 (Abb. 4b) die AfD-Bundessprecher*innen in einer deutlich bedrohlicheren Inszenierung. Alice Weidel und Alexander Gauland sind zusammen mit Bundeskanzlerin Angela Merkel (CDU) in den Parteifarben der AfD blau, rot und weiß ausgeleuchtet. Beschämt und schuldbewusst schaut Merkel zu Boden: Wohlwissend, so legt es zumindest die Inszenierung nahe, dass sie, stellvertretend für die Große Koalition, am Erfolg der AfD mitgewirkt hat. Deutlich größer und links hinter ihr schaut Weidel mit verschränkten Händen vor dem Kinn wie eine

\section{DER 5 PIFHEL}

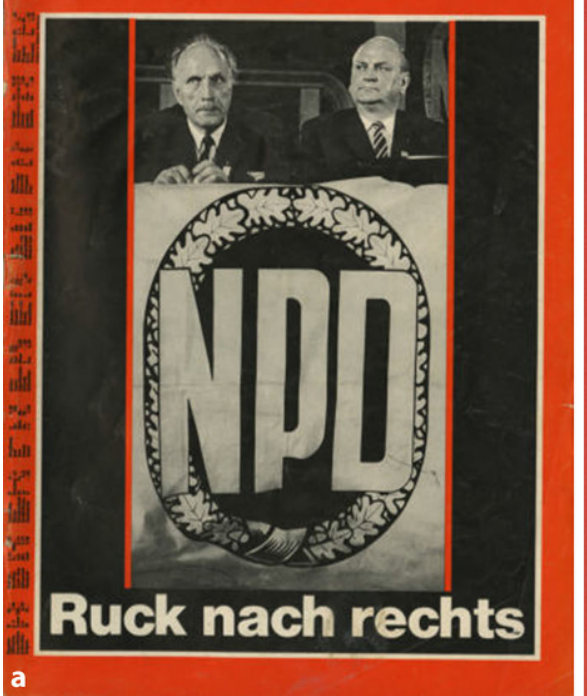

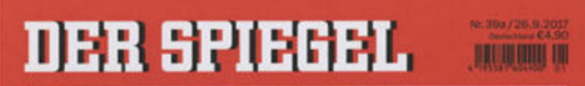

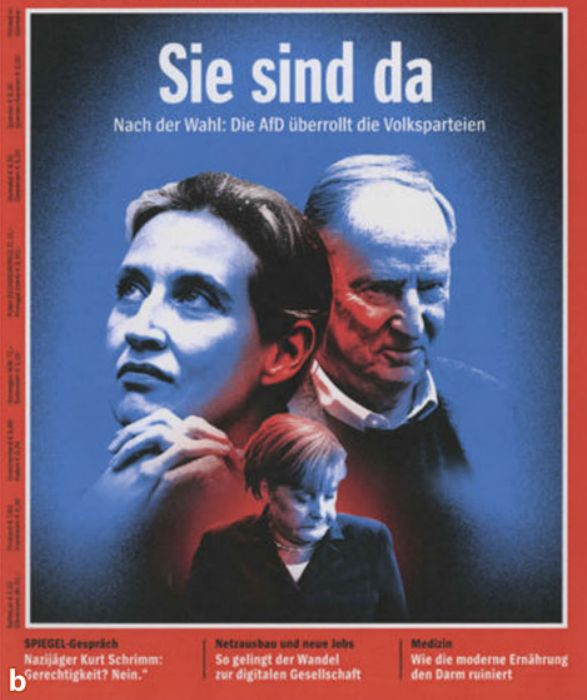

Abb. 4 a Der Spiegel vom 28. November 1966, Nr. 49; b Der Spiegel vom 26. September 2017, Nr. 39a 
Visionärin nach oben in die Ferne. Wiederum versetzt hinter Weidel blickt Gauland mit leicht geneigtem Kopf und angriffslustiger Miene am Betrachter vorbei. Kopfhaltung und Mimik korrespondieren mit den von ihm am Wahlabend geäußerten Worten: „Wir werden Frau Merkel jagen“ (zit. nach Diehl 2018a, S. 89). Die fokussierte Beleuchtung und die starken Farbkontraste, die dem Bild einer Wärmebildkamera ähneln, verstärken die bedrohliche Atmosphäre. Durch die vertikale Anordnung, die größere Darstellung und die erhöhte Position der AfD-Politiker*innen gegenüber Merkel entsteht der Eindruck von Dominanz (Schwartz 1981, S. 46 ff.). Das Wort ,überrollen“ unterstreicht dies, indem es die bildliche Assoziation des Rades bzw. des ,unter die Räder Kommens“ hervorruft. So setzt das Bild den visuellen Frame einer Übermacht der AfD vis-à-vis den etablierten Parteien, wenngleich den 12,6\% der Wählerstimmen für die AfD 26,8\% für die CDU und 20,5\% für die SPD gegenüberstanden.

Mit dem Titel „Sie sind da“ wird auf den 2015 verfilmten Roman Er ist wieder da von Timur Vermes (2012) angespielt. In der Satire, die Hitler zum Leben erwachen lässt, nimmt sich eine Agentur dem aus der Zeit gefallenen und deshalb zusehends verwirrten Diktator an, organisiert Fernsehauftritte und eine eigene TV-Show für ihn. Im Roman wird die Faszination für Hitler und die damit verbundene Aufmerksamkeitslogik der Massenmedien thematisiert. Gleichzeitig nutzt das Buchcover selbst die Popularität der Hitlerreferenz, indem es den schwarzen Seitenscheitel gekoppelt mit dem ikonischen Hitlerbart zeigt (Weinert 2018, S. $131 \mathrm{f}$.). Auch wenn auf dem Magazincover in Abb. 4b keine bildliche Referenz zu Hitler besteht, aktiviert die Überschrift den Bezug zum Roman und weckt damit die Assoziation zu Hitler.

Schließlich schafft die Überschrift gekoppelt mit der beschriebenen Vertikalität eine Dichotomie zwischen einem imaginierten „Uns“ und „,den Anderen“. Mit dem Wort „Sie“ werden Weidel und Gauland zu „den anderen“ bzw. zu Angehörigen einer out-group (Tajfel und Turner 1986). Das nicht explizit erwähnte „Wir“ bzw. die in-group sind die sogenannten etablierten Parteien und deren Anhänger*innen, zu denen potenziell auch die adressierte Leserschaft zählt. Diese Gruppe wird im Bild stellvertretend von der Kanzlerin verkörpert. Versteht man dies als Gegenüberstellung zwischen „Wir, die Demokraten“ und „Sie, die Rechtspopulisten“, scheint hier die normative Frage nach der Unterscheidung zwischen einem positiven Demokratie- und einem ausschließlich negativ konnotierten Populismusbegriff durch, die seit langem Teil der politikwissenschaftlichen Debatte ist (Canovan 1999; von Kielmansegg 2017, S. 272 ff.). Aus demokratietheoretischer Sicht bleibt auf die Problematik einer solchen Dichotomie hinzuweisen. Denn ,ihr Dasein“ ist im Sinne der repräsentativen Demokratie Ausdruck des Willens eines Teils der Wähler*innen.

\section{Fazit}

Mit Blick auf die Forschungslücke visueller Darstellungen rechtspopulistischer Akteure durch die Massenmedien untersucht die vorliegende Studie visuelle Frames in Bezug auf die AfD auf Titelbildern des Nachrichtenmagazins Der Spiegel und vergleicht diese mit Covern aus den Anfangsjahren der NPD. Unter dem theoretischen Blickwinkel der visuellen Politik wurde einleitend dafür plädiert, Bilder verstärkt 
zum Gegenstand sozialwissenschaftlicher Analyse zu machen, da sie unsere Vorstellungen von Politik entscheidend mitprägen. Um die möglichen Bildbedeutungen im Hinblick auf die NPD und die AfD herauszuarbeiten, wurde anschließend der ikonographisch-ikonologische Ansatz vorgestellt und auf das Material angewendet. Wenngleich die vorliegende Studie keine kausalen Verbindungen zwischen Medien und dem Erstarken der Parteien aufzuzeigen vermag, so legen die Coveranalysen im Sinne Herkmans ,underlying moral and normative confrontations“ (Herkman 2019, S. 253) in Bezug auf die beiden Parteien offen.

Zusammenfassend lässt sich festhalten, dass in den Titelbildern hinsichtlich beider Parteien häufig Bezüge zum Nationalismus und Nationalsozialismus salient sind. Zum einen zeigt sich dies in einer abstrakten und dynamischen Form, die über Symbole wie das Hakenkreuz, die Frakturschrift sowie die vertikale Verschmelzung der Parteilogos einen fließenden Übergang der historischen zur zeitgenössischen Gefahr von rechts nahelegt (Abb. 1), zum anderen in einer statischen und direkt gleichsetzenden Form, bei der Petry und Gauland in militärischer Pose auf dem Zeppelinfeld erscheinen (Abb. 2). Auch auf weiteren Covern wird auf visuelle wie textuelle Referenzen zurückgegriffen, die aus Geschichtsbüchern und Dokumentationen über die Zeit des Nationalsozialismus und von Nationalismus geprägten Phasen aus der deutschen Geschichte bekannt sind, wenn beispielsweise die Dynamik des Aufstiegs der Parteien im Mittelpunkt steht (Abb. 3a, b).

Die Nazi-Vergleiche können, insbesondere vor dem Hintergrund der assoziativen Logik visueller Kommunikation und ihrer emotionalen Dimension (Müller 1999, S. 19), als aufmerksamkeitssteigernde und damit lukrative mediale Strategie verstanden werden. Wenngleich die visuellen schematischen Analogien zum Nationalsozialismus nicht zwangsläufig gegeben sind (Abb. 4a), muss grundsätzlich auf die damit verbundenen Verzerrungen hingewiesen werden. So erscheinen die Vergleiche sowohl aus historischer Sicht als auch vor dem theoretischen Hintergrund visueller Politikvermittlung für den Diskurs in einer Demokratie diskussionswürdig. Sie bergen einerseits die Gefahr in sich, zur Verharmlosung der NS-Diktatur beizutragen. Andererseits kann eine Fokussierung auf diese Vergleiche zur Ablenkung von jeweils aktuellen politischen und gesellschaftlichen Debatten führen. So sind beispielsweise die in den Anfangsjahren zentrale Kritik der AfD an Modernisierungsund Globalisierungsprozessen sowie am Euro keine Titelthemen. Mit der Spendenaffäre Ende 2018 und den Verstrickungen zwischen der AfD und der russischen Regierung werden zumindest Bezüge zu aktuellen Ereignissen sichtbar. ${ }^{15}$

Exemplarisch werden in den hier betrachteten Covern Muster deutlich, die auch die textbasierte Berichterstattung etablierter Zeitungen über die AfD prägen: zum einen die Themensetzung und zum anderen das binäre Narrativ der Radikalen und der Gemäßigten innerhalb der Partei (Bey und Wamper 2018, S. 120 ff.). Der erste Aspekt bezieht sich auf das Aufgreifen bewusster Provokationen, wie z.B. Petrys Aussage des möglichen Schusswaffengebrauchs oder Gaulands Ankündigung einer Jagd auf Frau Merkel. Bernd Gäbler hat die heikle Frage nach dem medialen Umgang mit diesen gezielten Provokationen treffend als „Stöckchen-Spiel“ (Gäbler 2017) bezeichnet. Der zweite Aspekt lässt sich in abgewandelter Form anhand von

15 Diese sowie weitere in den Untersuchungszeitraum fallende Cover sind im Anhang verzeichnet. 
Abb. 4b illustrieren. Abgewandelt insofern, als darin nicht zwischen ,guten“ und „bösen“ AfD-Mitgliedern differenziert, sondern vielmehr eine Dichotomie zwischen Weidel und Gauland als pars pro toto für die AfD einerseits und den etablierten politischen Akteuren andererseits inszeniert wird. Damit eröffnet sich der Blick auf das Spannungsfeld zwischen Populismus und Demokratie. Die Frage, ob die AfD eine pathologische Form der Demokratie darstellt oder eine potentielle Korrekturfunktion übernimmt (von Kielmansegg 2017, S. $272 \mathrm{ff}$.) scheint auf diesem Titelbild zugunsten der pathologischen Form beantwortet zu werden.

Die vorliegende explorative Auseinandersetzung mit visuellen massenmedialen Darstellungen der AfD versteht sich als Ergänzung zu den Inhaltsanalysen der textuellen Medienberichterstattung über die Partei. Eine Ausweitung dieses neuen Forschungsfelds scheint aufgrund der weiter zunehmenden Radikalisierung der Partei und möglicher Entwicklungen im Verlauf ihres media life cycle (Mazzoleni 2008) geboten. ${ }^{16}$ Auch sind Studien zum visuellen Framing der AfD in Boulevardmedien wie z.B. der Bild wünschenswert, um Aufschluss über Unterschiede und Gemeinsamkeiten in den visuellen Deutungsmustern verschiedener Medienangebote geben zu können. Gleichzeitig müssen die medialen Visualisierungen der etablierten Parteien in den Blick genommen werden. Nur eine äquivalente Berücksichtigung von Medien- und Parteiendifferenzen kann Aufschluss darüber geben, ob bzw. in welchem Maß die vielerorts pauschal attestierte besondere Beziehung zwischen populistischen Akteuren und den Medien (Reinemann 2017, S. 177) existiert.

Aus methodischer Sicht bleibt festzuhalten, dass die ikonographisch-ikonologische Analyse für die Exploration visueller Medienframes gewinnbringend ist, da sie dem vermeintlich Offensichtlichen bewusst eine analytische Distanz vorschaltet und es erlaubt, Stück für Stück mögliche Bedeutungen zu rekonstruieren. Eine derart kleinschrittige, zunächst deskriptive Arbeit ist notwendig, um das Visuelle adäquat in einen Text umzuwandeln und theoretisch reflektieren zu können (Pilarczyk 2017, S. 83). Der hermeneutisch-interpretative Zugang erlaubt zwar keine unmittelbaren Aussagen zur Bildwirkung. Einschränkend bleibt zudem auf den Unterschied zwischen der detaillierten und zeitintensiven wissenschaftlichen Bildbetrachtung und den in der Regel nur kurzen Blicken der potentiellen Leser*innen hinzuweisen, die möglicherweise zu anderen Deutungen führen. Wenn man Magazincover jedoch als kulturelle Artefakte (Kitch 2018) begreift und die mediale Bildsprache weit auseinanderliegender Zeiträume kontrastieren sowie Details der Bildgestaltung methodisch angemessen reflektieren will, bleibt die ikonographisch-ikonologische Methode ein starkes Instrument.

Danksagung Ich bedanke mich bei zwei anonymen Gutachter*innen für ihre wertvollen Anmerkungen zum Manuskript. Mein Dank gilt zudem Horst-Alfred Heinrich, Michael Kohlstruck, Bernhard Stahl und Jannis Alberts für ihren kritischen Blick auf frühere Fassungen des Aufsatzes.

Funding Open Access funding provided by Projekt DEAL.

16 Zweifelsohne hat die AfD in den letzten Jahren personell wie ideologisch einen Wandel durchlebt. Zu den aktuellen Entwicklungen gehören unter anderem die Regierungskrise in Thüringen, die Auflösung des völkischen Flügels sowie die Diskussion um die Parteimitgliedschaft von Andreas Kalbitz. Siehe hierzu Abb. 10 und Abb. 11 im Anhang. 
Open Access Dieser Artikel wird unter der Creative Commons Namensnennung 4.0 International Lizenz veröffentlicht, welche die Nutzung, Vervielfältigung, Bearbeitung, Verbreitung und Wiedergabe in jeglichem Medium und Format erlaubt, sofern Sie den/die ursprünglichen Autor(en) und die Quelle ordnungsgemäß nennen, einen Link zur Creative Commons Lizenz beifügen und angeben, ob Änderungen vorgenommen wurden.

Die in diesem Artikel enthaltenen Bilder und sonstiges Drittmaterial unterliegen ebenfalls der genannten Creative Commons Lizenz, sofern sich aus der Abbildungslegende nichts anderes ergibt. Sofern das betreffende Material nicht unter der genannten Creative Commons Lizenz steht und die betreffende Handlung nicht nach gesetzlichen Vorschriften erlaubt ist, ist für die oben aufgeführten Weiterverwendungen des Materials die Einwilligung des jeweiligen Rechteinhabers einzuholen.

Weitere Details zur Lizenz entnehmen Sie bitte der Lizenzinformation auf http://creativecommons.org/ licenses/by/4.0/deed.de.

\section{Anhang}

\section{Weitere Titelbilder}

Abb. 5, 6, 7, 8, 9, 10 und 11 .

Abb. 5 Der Spiegel vom 21. Oktober 1968, Nr. 43

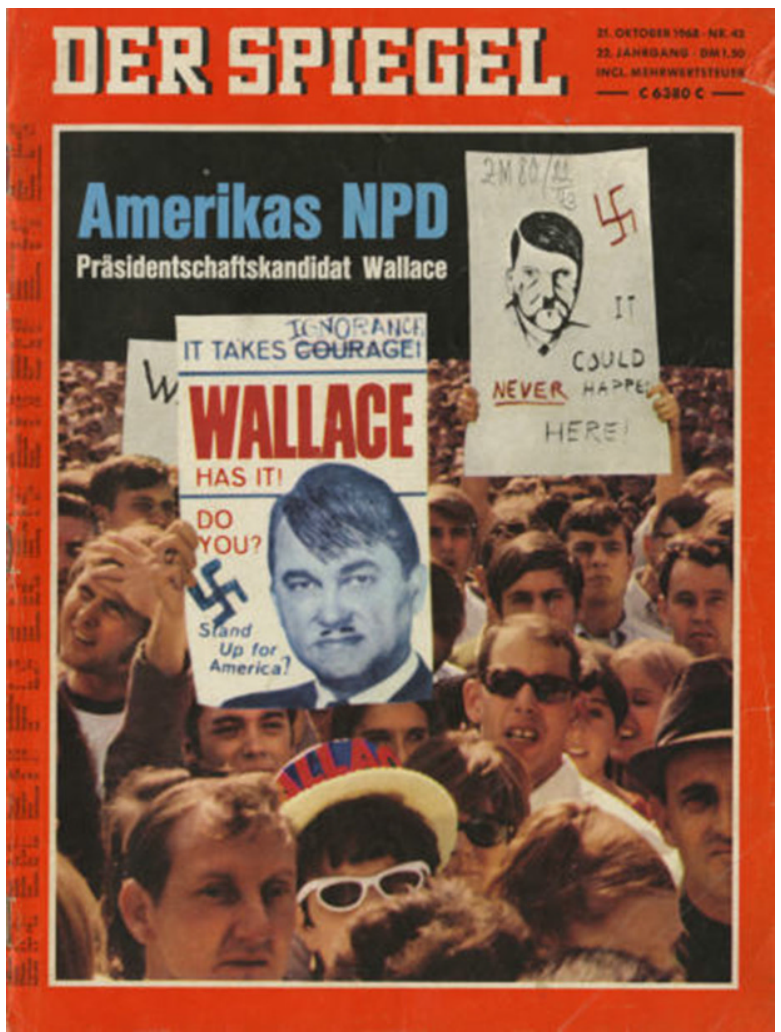


Abb. 6 Der Spiegel vom 23. Dezember 1968, Nr. 52

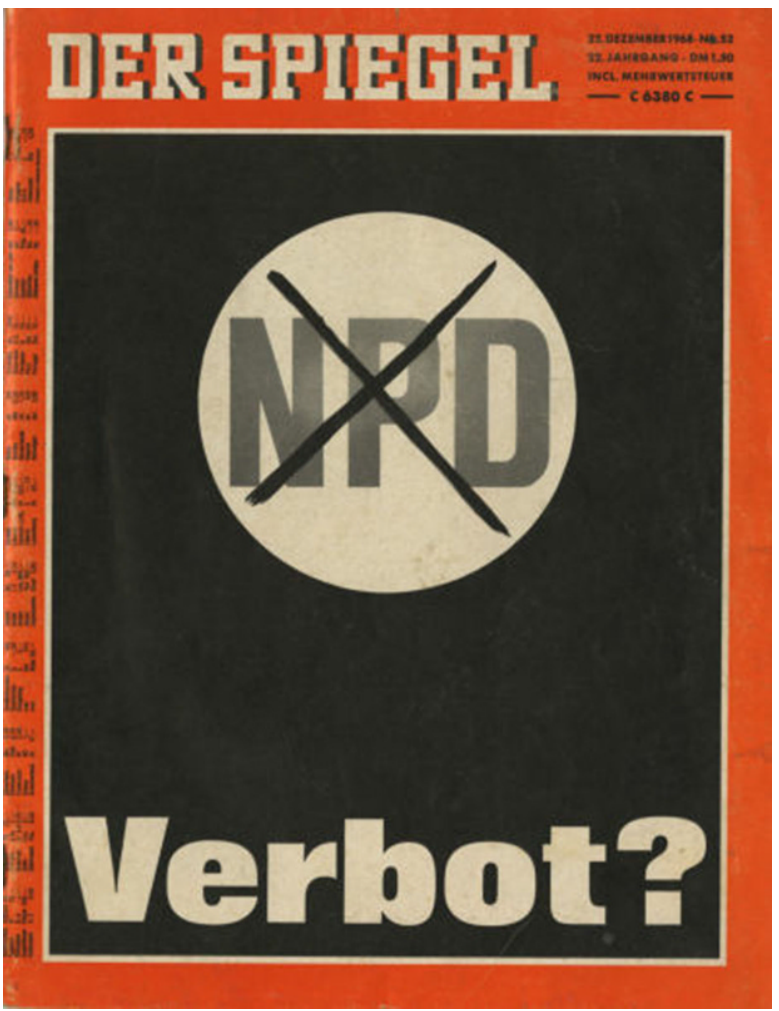


Abb. 7 Der Spiegel vom 24. November 2018, Nr. 48

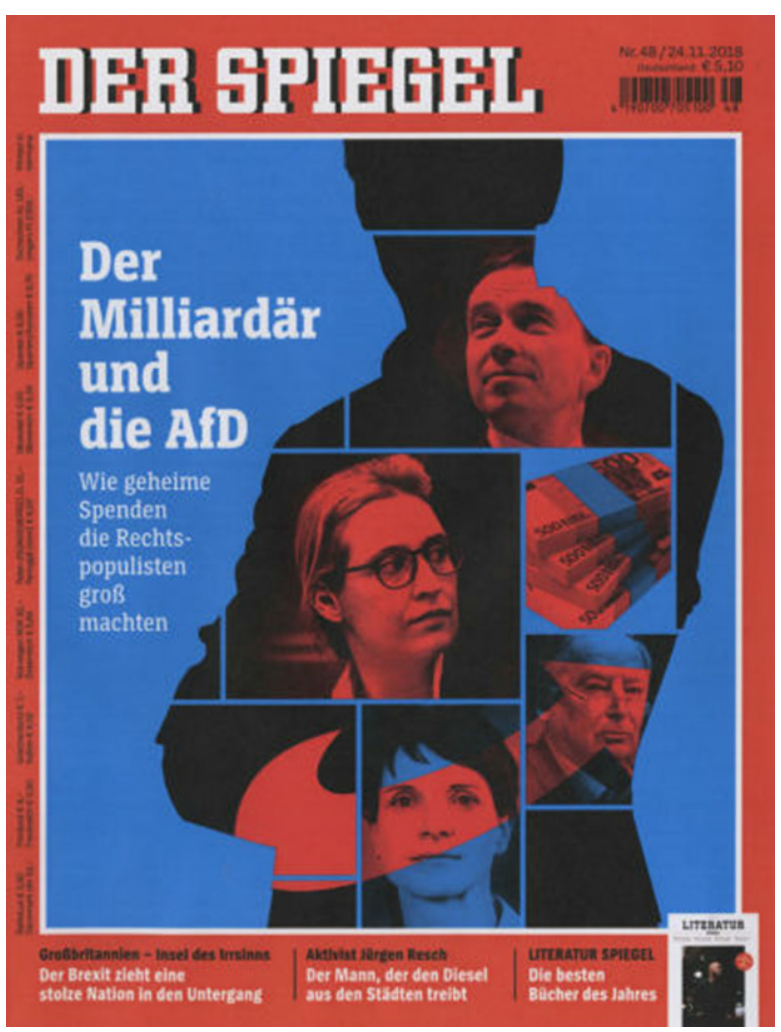


Abb. 8 Der Spiegel vom 6. April 2019, Nr. 15

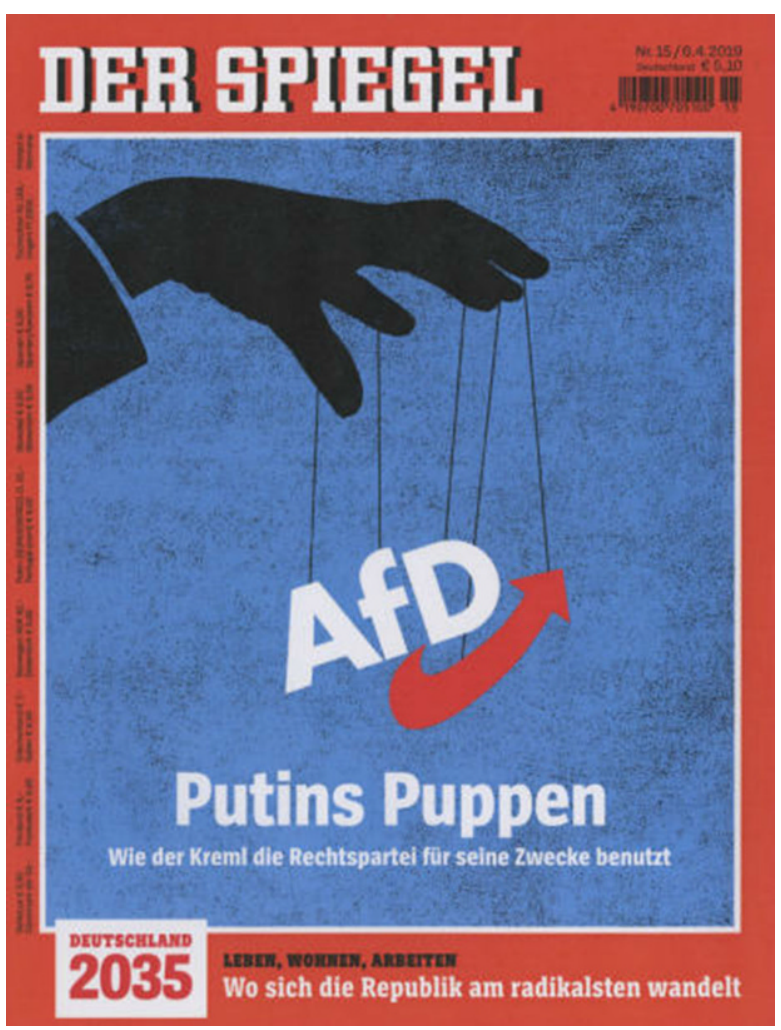


Abb. 9 Der Spiegel vom 25. Mai 2019, Nr. 22

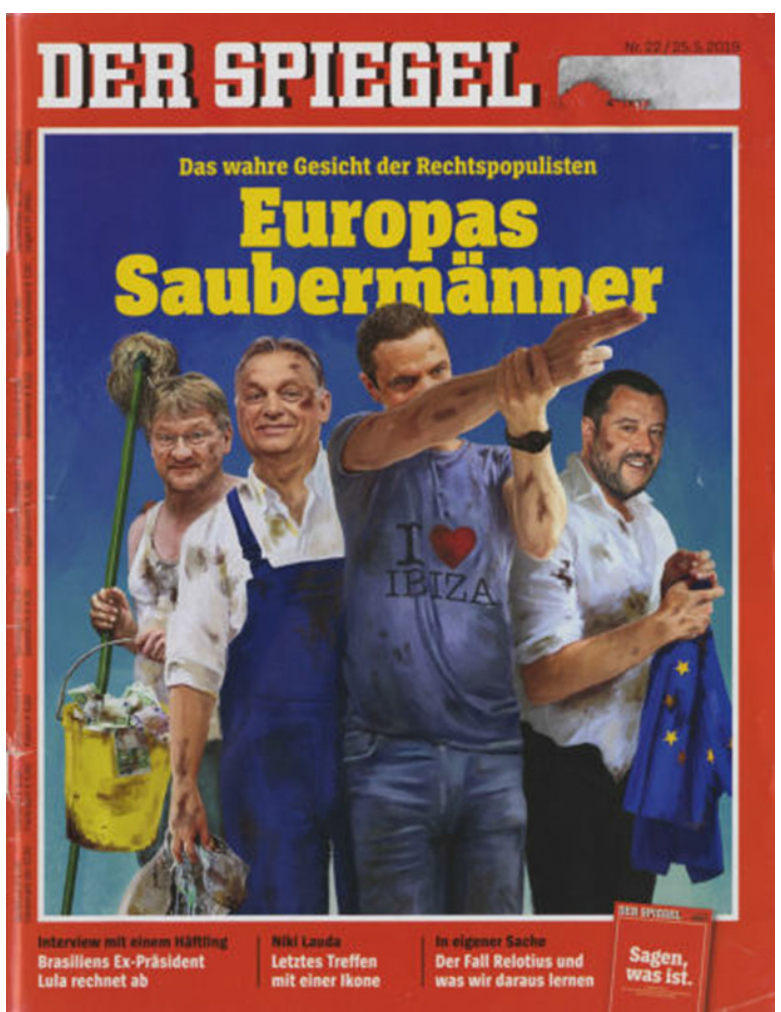


Abb. 10 Der Spiegel vom 8. Februar 2020, Nr. 7
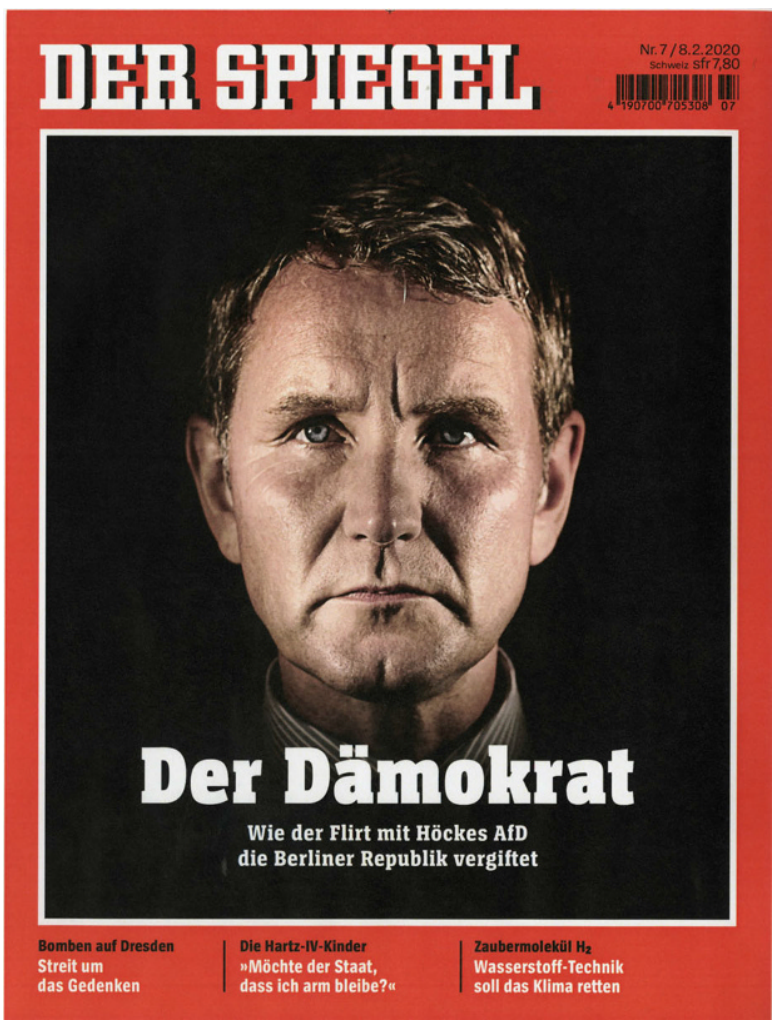
Abb. 11 Der Spiegel vom 23. Mai 2020, Nr. 22

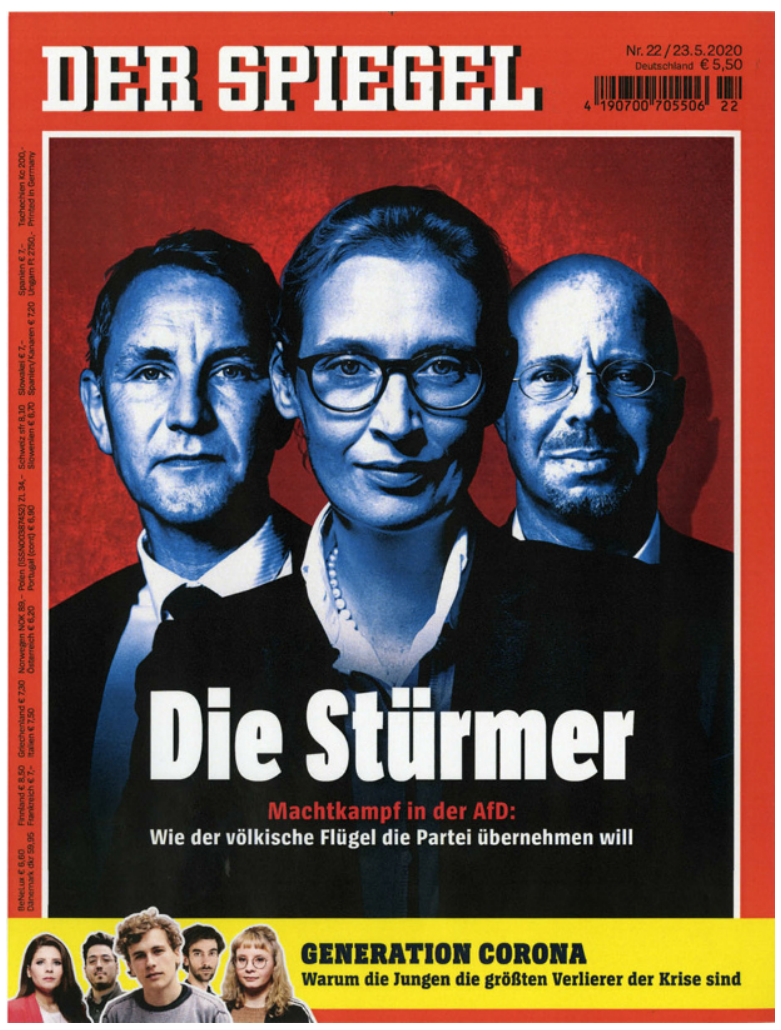




\section{Literatur}

Aalberg, Toril, Frank Esser, Carsten Reinemann, Jesper Strömbäck, und Claes de Vreese (Hrsg.). 2017. Populist political communication in Europe. New York: Routledge.

Aiello, Giorgia, und Katy Parry. 2020. Visual communication. Understanding images in media culture. London: SAGE.

Arzheimer, Kai. 2015. The AfD: finally a successful right-wing populist eurosceptic party for Germany? West European Politics 38(3):535-556.

Bey, Paul, und Regina Wamper. 2018. Die „Mainstream-Medien“: Stütze der AfD? In Völkisch-autoritärer Populismus. Der Rechtsruck in Deutschland und die AfD, Hrsg. Alexander Häusler, 117-125. Hamburg: VSA.

Bourdieu, Pierre. 1997. Der Tote packt den Lebenden. Hamburg: VSA.

Bowman, J.A. 1989. Line infantry. The Pickelhaube, Bd. 1. Lancaster: Imperial Publications.

Bredekamp, Horst. 2020. Thomas Hobbes Der Leviathan. Das Urbild des modernen Staates und seine Gegenbilder - 1651-2001. Berlin: De Gruyter.

Canovan, Margaret. 1999. Trust the people! populism and the two faces of democracy. Political Studies 47:2-16.

Coles, Anthony. 2014. John Heartfield. Ein politisches Leben. Köln: Böhlau.

Dahinden, Urs. 2006. Framing. Eine integrative Theorie der Massenkommunikation. Konstanz: UVK.

Dahrendorf, Ralf. 1968. Das Ende eines Wunders. In Die Restauration entläßt ihre Kinder oder Der Erfolg der Rechten in der Bundesrepublik, Hrsg. Freimut Duve, 90-96. Reinbek: Rowohlt.

Decker, Frank, und Marcel Lewandowsky. 2017. Rechtspopulismus in Europa: Erscheinungsformen, Ursachen und Gegenstrategien. Zeitschrift für Politik 64(1):21-38.

Der Spiegel. 1966. Wotans Wähler. Der Spiegel, Bd. 15, 30-40.

Der Spiegel. 1967. Rechts ab zum Vaterland. Der Spiegel, Bd. 17, 72-93.

Der Spiegel. 1968. NPD. Tut und tut. Der Spiegel, Bd. 52, 25-31.

Der Spiegel. 2016. Im Schützengraben. Der Spiegel, Bd. 6, 12-20.

Diehl, Paula. 2018a. Rechtspopulismus und Massenmedien. Eine explosive Mischung. In Großerzählungen des Extremen. Neue Rechte, Populismus, Islamismus, War on Terror, Hrsg. Jennifer Schellhöh, Jo Reichertz, Volker M. Heins, und Armin Flender, 87-96. Bielefeld: transcript.

Diehl, Paula. 2018b. Die Symbolisierung des Volkes in der Demokratie: Eine ikonografische Spurensuche. Leviathan 46(Sonderband 34):23-47.

Donges, Patrick, und Otfried Jarren. 2017. Politische Kommunikation in der Mediengesellschaft. Eine Einführung. Wiesbaden: Springer VS.

Drechsel, Benjamin. 2009. Trügerischer Augenschein. Hinweise zur Verflechtung von politischer Kultur und visueller Politik. In Politische Kultur. Forschungsstand und Forschungsperspektiven, Hrsg. Samuel Salzborn, 147-174. Frankfurt a.M.: Peter Lang.

Dudek, Peter, und Hans-Gerd Jaschke. 1984. Entstehung und Entwicklung des Rechtsextremismus in der Bundesrepublik. Zur Tradition einer besonderen politischen Kultur. Bd. 1. Opladen: Westdeutscher Verlag.

Eitz, Thorsten, und Georg Stötzel. 2009. Diskurs über NS-Vergleiche. In Wörterbuch der „Vergangenheitsbewältigung “. Die NS-Vergangenheit im öffentlichen Sprachgebrauch, Bd. 2, Hrsg. Thorsten Eitz, Georg Stötzel, 12-38. Hildesheim: Georg Olms.

Entman, Robert, Jörg Matthes, und Lynn Pellicano. 2009. Nature, sources, and effects of news framing. In The handbook of journalism studies, Hrsg. Karin Wahl-Jorgensen, Thomas Hanitzsch, 175-190. New York: Routledge.

Fetscher, Iring. 1967. Rechtes und rechtsradikales Denken in der Bundesrepublik. In Rechtsradikalismus, Hrsg. Iring Fetscher, 11-29. Frankfurt a.M.: Europäische Verlagsanstalt.

Franzmann, Simon T., und Marcel Lewandowsky. 2020. Populismus? Populismen! Programmatische Heterogenität rechtspopulistischer Parteien in Westeuropa. Bonn: Bonner Akademie für Forschung und Lehre praktischer Politik (BAPP).

Frederik, Hans. 1966. NPD. Gefahr von rechts? München: Verlag Politisches Archiv.

Fuhrmann, Horst. 1992. Pour le mérite. Über die Sichtbarmachung von Verdiensten. Eine historische Besinnung. Sigmaringen: Thorbecke.

Funcken, Liliane, und Fred Funcken. 1983. Historische Uniformen. 19. Jahrhundert. 1850-1900: Frankreich, Großbritannien, Deutschland, Österreich, Rußland. Infanterie, Kavallerie, technische Truppen, Artillerie. München: Mosaik.

Gäbler, Bernd. 2017. OBS-Arbeitsheft 92. AfD und Medien. Analyse und Handreichungen. Frankfurt a.M.: Otto Brenner Stiftung. 
Gäbler, Bernd. 2018. OBS-Arbeitsheft 95. AfD und Medien. Erfahrungen und Lehren für die Praxis. Frankfurt a.M.: Otto Brenner Stiftung.

Gadinger, Frank, und Elena Simon. 2019. Kalkulierte Ambivalenz, mobilisierte Ängste und volksnahe Inszenierung: Rechtspopulistische Erzählstrategien in Wahlkampagnen und Regierungspraxis. Zeitschrift für Politikwissenschaft 29(1):23-52.

Geise, Stephanie, und Christian Baden. 2015. Putting the image back into the frame: modeling the linkage between visual communication and frame-processing theory. Communication Theory 25(1):46-69.

Geise, Stephanie, Katharina Lobinger, und Cornelia Brantner. 2013. Fractured Paradigm? Theorien, Konzepte und Methoden der visuellen Framingforschung: Ergebnisse einer systematischen Literaturschau. In Visual Framing. Perspektiven und Herausforderungen der Visuellen Kommunikationsforschung, Hrsg. Stephanie Geise, Katharina Lobinger, 42-76. Köln: von Halem.

Genette, Gérard. 2001. Paratexte. Das Buch vom Beiwerk des Buches. Frankfurt a.M.: Suhrkamp.

Gerth, Sebastian. 2012. Die Erweiterte Bildtypenanalyse. Ein Instrument zur Untersuchung von Pressefotografien aus Rezipienten- und Medienperspektive. In Bilder - Kulturen - Identitäten. Analysen zu einem Spannungsfeld Visueller Kommunikationsforschung, Hrsg. Stephanie Geise, Katharina Lobinger, 285-308. Köln: von Halem.

Goffman, Erving. 1974. Frame analysis: an essay on the organization of experience. Boston: Northeastern University Press.

Grass, Günter. 1959. Die Blechtrommel. Darmstadt: Luchterhand.

Herbert, Ulrich. 2015. Der alte neue Diktator. DIE ZEIT 50:57.

Herkman, Juha. 2016. Construction of populism. Meanings given to populism in the nordic press. Nordicom Review 37(Special Issue):147-161.

Herkman, Juha. 2019. Populism in political cartoons: caricatures of Nordic populist leaders. Popular Communication 17(3):252-267.

Hofmann, Wilhelm (Hrsg.). 1999a. Die Sichtbarkeit der Macht. Theoretische und empirische Untersuchungen zur visuellen Politik. Baden-Baden: Nomos.

Hofmann, Wilhelm. 1999b. Die Sichtbarkeit der Macht. Überlegungen zum Paradigmenwechsel von der „logozentrischen“ zur ,ikonozentrischen“ Politik. In Die Sichtbarkeit der Macht. Theoretische und empirische Untersuchungen zur visuellen Politik, Hrsg. Wilhelm Hofmann, 7-11. Baden-Baden: Nomos.

Hofmann, Wilhelm. 2009. „Ich schau Dir in die Augen“: Die Bedeutung visueller Medien für die politische Kommunikation in entwickelten Demokratien. In Strategien der Visualisierung. Verbildlichung als Mittel politischer Kommunikation, Hrsg. Herfried Münkler, Jens Hacke, 109-125. Frankfurt a.M.: Campus.

Humburg, Norbert. 1990. er Rattenfänger von Hameln. Die berühmte Sagengestalt in Geschichte und Literatur, Malerei und Musik, auf der Bühne und im Film. Hameln: CW Niemeyer.

IVW. 2019. Quartalsauflagen. https://www.ivw.eu/print/quartalsauflagen/quartalsauflagen. Zugegriffen: 16. März 2020 .

Jaschke, Hans-Gerd. 2016. Strategien der extremen Rechten in Deutschland nach 1945. In Handbuch Rechtsextremismus, Hrsg. Fabian Virchow, Martin Langebach, und Alexander Häusler, 115-134. Wiesbaden: Springer VS.

Jesse, Eckhard, und Isabelle-Christine Panreck. 2017. Populismus und Extremismus. Terminologische Abgrenzung - das Beispiel der AfD. Zeitschrift für Politik 64(1):59-76.

Kanter, Heike. 2016. Ästhetisches Agieren und die Auslegung von Welt - ikonische Macht in der Gestaltung von Pressefotografien. Österreichische Zeitschrift für Soziologie 41:187-211.

Kappas, Arvid, und Marion Müller. 2006. Bild und Emotion - ein neues Forschungsfeld. Theoretische Ansätze aus Emotionspsychologie, Bildwissenschaft und visueller Kommunikationsforschung. Publizistik 51(1):3-23.

Keunecke, Hans-Otto. 1993. Die deutsche Schrift im Dritten Reich. Die Nationalsozialisten und das Schicksal der gebrochenen Lettern. Buchhandelsgeschichte: Aufsätze, Rezensionen und Berichte zur Geschichte des Buchwesens 1993/4:B121-B129.

Graf von Kielmansegg, Peter. 2017. Demos und Populus: Demokratietheoretische Annäherungen an das Phänomen Populismus. In Populismus. Varianten von Volksherrschaft in Geschichte und Gegenwart, Hrsg. Thorsten Beigel, Georg Eckert, 267-281. Münster: Aschendorff.

Kitch, Carolyn. 2018. Theory and methods of analysis. Models for understanding magazines. In The Routledge handbook of magazine research. The future of the magazine form, Hrsg. David Abrahamson, Marcia Prior-Miller, 9-21. New York: Routledge.

Klietmann, Kurt-Georg. 2004. Auszeichnungen des Deutschen Reiches 1936-1945. Eine Dokumentation ziviler und militärischer Verdienst- und Ehrenzeichen. Stuttgart: Motorbuch. 
Korte, Karl-Rudolf (Hrsg.). 2015. Emotionen und Politik. Begründungen, Konzeptionen und Praxisfelder einer politikwissenschaftlichen Emotionsforschung. Baden-Baden: Nomos.

Kühnl, Reinhard. 1967. Die NPD. Struktur, Programm und Ideologie einer neofaschistischen Partei. Berlin: Voltaire.

Ludwig, Roisin, und Regina Wamper. 2017. Schnittstellen und Abgrenzungen. Zum Umgang der FAZ mit der populistischen extremen Rechten. In Autoritäre Zuspitzung. Rechtsruck in Europa, Hrsg. Isolde Aigner, Paul Jobst, und Regina Wamper, 95-120. Münster: Unrast.

Manow, Philip, Friedbert W. Rüb, und Dagmar Simon (Hrsg.). 2012. Die Bilder des Leviathan. Eine Deutungsgeschichte. Baden-Baden: Nomos.

Marcinkowski, Frank (Hrsg.). 2014. Framing als politischer Prozess. Beiträge zum Deutungskampf in der politischen Kommunikation. Baden-Baden: Nomos.

Marotzki, Winfried, und Katja Stoetzer. 2006. Die Geschichten hinter den Bildern. Annäherungen an eine Methode und Methodologie der Bildinterpretation in biographie- und bildungstheoretischer Absicht. In Bildinterpretation und Bildverstehen. Methodische Ansätze aus sozialwissenschaftlicher, kunstund medienpädagogischer Perspektive, Hrsg. Winfried Marotzki, Horst Niesyto, 15-44. Wiesbaden: Springer VS.

Matthes, Jörg. 2008. Medien-Frames inhaltsanalytisch (be)greifen. Eine Analyse von 135 nationalen und internationalen Fachzeitschriftenaufsätzen, 1990-2005. In Die Brücke zwischen Theorie und Empirie: Operationalisierung, Messung und Validierung in der Kommunikationswissenschaft, Hrsg. Jörg Matthes, Werner Wirth, und Andreas Fahr, 157-177. Köln: von Halem.

Mazzoleni, Gianpietro. 2008. Populism and the media. In Twenty-first century populism. The spectre of western European democracy, Hrsg. Daniele Albertazzi, Duncan McDonnell, 49-62. Basingstoke: Palgrave Macmillan.

Miller-Idriss, Cynthia. 2017. The extreme gone mainstream. Commercialization and far right youth culture in Germany. Princeton: Princeton University Press.

Mogge, Winfried. 2007. „Und heute gehört uns Deutschland...“. Entstehung und Nachwirkungen eines Liedes 1933-1993. In Good-bye memories? Lieder im Generationengedächtnis des 20. Jahrhunderts, Hrsg. Barbara Stambolis, Jürgen Reulecke, 175-184. Essen: Klartext.

Müller, Marion. 1999. Politische Vision. In Die Sichtbarkeit der Macht. Theoretische und empirische Untersuchungen zur visuellen Politik, Hrsg. Wilhelm Hofmann, 15-27. Baden-Baden: Nomos.

Müller, Marion. 2011. Ikonografie und Ikonologie, visuelle Kontextanalyse, visuelles Framing. In Die Entschlüsselung der Bilder. Methoden zur Erforschung visueller Kommunikation, Hrsg. Thomas Petersen, Clemens Schwender, 28-55. Köln: von Halem.

Müller, Marion, und Stephanie Geise. 2015. Grundlagen der visuellen Kommunikation. Konstanz: UVK.

Münkler, Herfried, und Jens Hacke (Hrsg.). 2009. Strategien der Visualisierung. Verbildlichung als Mittel politischer Kommunikation. Frankfurt a.M.: Campus.

Niethammer, Lutz. 1969. Angepaßter Faschismus. Politische Praxis der NPD. Frankfurt a.M.: Fischer.

Panofsky, Erwin. 1975. Sinn und Deutung in der bildenden Kunst. Köln: Dumont.

Paul, Gerhard. 2008. Das Jahrhundert der Bilder: 1949 bis heute. Göttingen: Vandenhoeck \& Ruprecht.

Pfahl-Traughber, Armin. 2019. Rechtsextremismus in Deutschland. Eine kritische Bestandsaufnahme. Wiesbaden: Springer VS.

Pilarczyk, Ulrike. 2017. Grundlagen der seriell-ikonografischen Fotoanalyse. Jüdische Jugendfotografie in der Weimarer Zeit. In Visual History: Bilder und Bildpraxen in der Geschichte, Bd. 3, Hrsg. Jürgen Danyel, Gerhard Paul, und Annette Vowinckel, 75-99. Göttingen: Wallstein.

Potthoff, Matthias. 2012. Medien Frames und ihre Entstehung. Wiesbaden: Springer VS.

Priester, Karin. 2016. Rechtspopulismus - ein umstrittenes theoretisches und politisches Phänomen. In Handbuch Rechtsextremismus, Hrsg. Fabian Virchow, Martin Langebach, und Alexander Häusler, 533-560. Wiesbaden: Springer VS.

Ramachandran, Vilayanur, und William Hirstein. 1999. The science of Art. A neurological theory of aesthetic experience. Journal of Consciousness Studies 6(6-7):15-51.

Reinemann, Carsten. 2017. Populismus, Kommunikation, Medien. Ein Überblick über die Forschung zu populistischer politischer Kommunikation. Zeitschrift für Politik 64(2):167-190.

Rohe, Karl. 1987. Politische Kultur und der kulturelle Aspekt von politischer Wirklichkeit. Konzeptionelle und typologische Überlegungen zu Gegenstand und Fragestellung Politischer Kultur-Forschung. Politische Vierteljahresschrift 28(Sonderheft 18):39-48.

Rössler, Patrick. 2014. Visuelles Framing durch Neue Typographie: Eine historische Inhaltsanalyse. In Framing als politischer Prozess. Beiträge zum Deutungskampf in der politischen Kommunikation, Hrsg. Frank Marcinkowski, 45-61. Baden-Baden: Nomos. 
Rummens, Stefan. 2017. Populism as a threat to liberal democracy. In The Oxford handbook of populism, Hrsg. Cristóbal Rovira Kaltwasser, Paul Taggart, und Paulina Ochoa Espejo, 554-570. Oxford: Oxford University Press.

Sarcinelli, Ulrich. 1989. Symbolische Politik und politische Kultur. Das Kommunikationsritual als politische Wirklichkeit. Politische Vierteljahresschrift 30(2):292-309.

Sarcinelli, Ulrich. 2011. Politische Kommunikation in Deutschland. Zur Politikvermittlung im demokratischen System. Wiesbaden: Springer VS.

Schlürmann, Jan. 2015. Die „Wirmer-Flagge“. Die wechselhafte Geschichte eines vergessenen Symbols der deutschen Christlichen Demokratie. Historisch-Politische Mitteilungen 22(1):331-342.

Schütt, Hans-Dieter, und Oliver Schwarzkopf (Hrsg.). 1999. Die SPIEGEL-Titelbilder 1947-1999. Berlin: Schwarzkopf \& Schwarzkopf.

Schwartz, Barry. 1981. Vertical classification. A study in structuralism and the sociology of knowledge. Chicago: University of Chicago Press.

Semetko, Holli A. 2007. Political communication. In The Oxford handbook of political behaviour, Hrsg. Russell J. Dalton, Hans-Dieter Klingemann, 123-143. Oxford: Oxford University Press.

von Sikorski, Christian, und Mark Ludwig. 2018. The effects of visual isolation on the perception of scandalized politicians. Communications 43(2):235-257.

Spiker, Ted. 2018. The magazine cover. The craft of identity and impact. In The Routledge handbook of magazine research. The future of the magazine form, Hrsg. David Abrahamson, Marcia Prior-Miller, 377-391. New York: Routledge.

Steuwer, Janosch. 2017. Das „Volk“ vorzeigen: Die Reichsparteitage und die populistische Legitimation des Nationalsozialismus. In Populismus. Varianten von Volksherrschaft in Geschichte und Gegenwart, Hrsg. Thorsten Beigel, Georg Ecker, 191-206. Münster: Aschendorff.

Stier, Sebastian, Lisa Posch, Arnim Bleier, und Markus Strohmaier. 2017. When populists become popular: comparing Facebook use by the right-wing movement Pegida and German political parties. Information, Communication \& Society 20(9):1365-1388.

Tajfel, Henri, und John Turner. 1986. The social identity theory of intergroup behavior. In Psychology of intergroup relations, Hrsg. Stephen Worchel, William Austin, 7-24. Chicago: Nelson-Hall.

Ulrich, Anne. 2017. Mehr Demokratie kommt nicht von rechts. Blätter für deutsche und internationale Politik 62(10):13-16.

Veneti, Anastasia, Daniel Jackson, und Darren Lilleker. 2019. Visual political communication. Cham: Palgrave Macmillan.

Vermes, Timur. 2012. Er ist wieder da. Köln: Eichborn.

Vorländer, Hans, Maik Herold, und Steven Schäller. 2018. PEGIDA and new right-wing populism in Germany. Cham: Springer Nature.

Weinert, Frederik. 2018. Nazi-Vergleiche und Political Correctness. Eine sprach- und kommunikationswissenschaftiche Analyse. Baden-Baden: Nomos.

Werz, Nikolaus. 2013. Erscheinungsformen und Debatten um den „Populismus“ in Deutschland. In Deutsche Kontroversen. Festschrift für Eckhard Jesse, Hrsg. Alexander Gallus, Thomas Schubert, und Tom Thieme, 421-435. Baden-Baden: Nomos.

Yanow, Dvora. 2014. Neither rigorous nor objective? Interrogating criteria for knowledge claims in interpretive science. In Interpretation and method. Empirical research methods and the interpretive turn, Hrsg. Dvora Yanow, Peregrine Schwartz-Shea, 97-119. New York: M. E. Sharpe. 